\title{
Fractional control of heat diffusion systems
}

\author{
Isabel S. Jesus • J.A. TenreiroMachado
}

\begin{abstract}
The concept of differentiation and integration to non-integer order has its origins in the seventeen century. However, only in the second-half of the twenty century appeared the first applications related to the area of control theory. In this paper we consider the study of a heat diffusion system based on the application of the fractional calculus concepts. In this perspective, several control methodologies are investigated and compared. Simulations are presented assessing the performance of the proposed fractional-order algorithms.
\end{abstract}

Keywords PID tuning · Fractional calculus ·

Fractional-order systems · ISE and ITSE optimization

\section{Introduction}

Fractional calculus (FC) is a generalization of integration and differentiation to a noninteger order $\alpha \in \mathrm{C}$, being the fundamental operator $a D_{t}^{\alpha}$, where $a$ and $t$ are the limits of the operation $[1,2]$. The FC concepts constitute a useful tool to describe several physical phenomena, such as heat, flow, electricity, magnetism, mechanics, or fluid dynamics. Presently, the FC theory is applied in almost all areas of science and engineering; its ability being recognized in improving the modeling and control of many dynamical systems. In fact, during the last years, $\mathrm{FC}$ has been used increasingly to model the constitutive behavior of materials and physical systems exhibiting hereditary and memory properties. This is the main advantage of fractional-order derivatives in comparison with classical integer-order models, where these effects are simply neglected.

It is well known that the fractional-order operator $s^{0.5}$ appears in several types of problems [3]. The transmission lines, the heat flow, or the diffusion of neutrons in a nuclear reactor are examples where the half-operator is the fundamental element. Moreover, diffusion is one of the three fundamental partial differential equations of mathematical physics [4]. Therefore, the control of such systems having in mind FC concepts is an important subject.

In this paper, we investigate several control strategies for the heat diffusion system based on fractionalorder algorithms. The fractional-order PID controller $\left(\mathrm{PI}^{\alpha} \mathrm{D}^{\beta}\right.$ controller) involves an integrator of order $\alpha \in \mathrm{m}^{+}$and a differentiator of order $\beta \in \mathrm{m}^{+}$. The good performance of this type of controller was demonstrated, in comparison with the conventional PID algorithms.

Bearing these ideas in mind, the paper is organized as follows. Section 2 gives the fundamentals of fractional-order control systems. Section 3 introduces 
the heat diffusion system and describes its simulation. Section 4 studies several control strategies and discusses the results. Finally, Sect. 5 draws the main conclusions and addresses perspectives toward future developments.

\section{Fractional-order control systems}

Fractional-order control systems are characterized by differential equations that have in the dynamical system and/or in the control algorithm, an integral and/or a derivative of fractional-order. Due to the fact that these operators are defined by irrational continuous transfer functions, in the Laplace domain, or infinite dimensional discrete transfer functions, in the $Z$ domain, we often encounter evaluation problems in the simulations. Therefore, when analyzing fractionalorder systems, we usually adopt continuous or discrete integer-order approximations of fractional-orderoperators $[5,8]$. The following two subsections provide a background for the remaining of the article by giving the fundamental aspects of the FC, and the discrete integer-order approximations of fractional-orderoperators.

\subsection{Fundamentals of fractional calculus}

The mathematical definition of a fractional-order derivative and integral has been the subject of several different approaches $[1,2,9,10]$. One commonly used definition for the fractional-order derivative is given by the Riemann-Liouville definition $(\alpha>0)$ : ${ }_{a} D^{\alpha}$

$$
\begin{aligned}
{ }_{a} D_{t}^{\alpha} f(t) & =\frac{1}{\Gamma(n-\alpha)} \frac{d^{n}}{d t^{n}} \int_{a}^{t} \frac{f(\tau)}{(t-\tau)^{\alpha-n+1}} d \tau, \\
n-1 & <\alpha<n,
\end{aligned}
$$

where $f(t)$ is the applied function and $r(x)$ is the Gamma function of $x$. Another widely used definition is given by the Grünwald-Letnikov approach $(\alpha \in \mathrm{m})$ :

$$
\begin{aligned}
& { }_{a} D_{t}^{\alpha} f(t)=\lim _{h \rightarrow 0} \frac{1}{h^{\alpha}} \sum_{k=0}^{\left[\frac{t-a}{h}\right]}(-1)^{k}\left(\begin{array}{l}
\alpha \\
k
\end{array}\right) f(t-k h), \\
& \left(\begin{array}{l}
\alpha \\
k
\end{array}\right)=\frac{\Gamma(\alpha+1)}{\Gamma(k+1) \Gamma(\alpha-k+1)},
\end{aligned}
$$

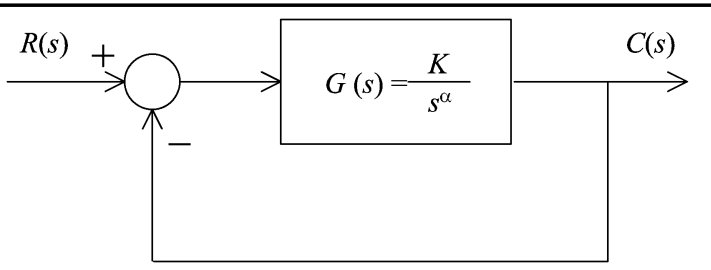

Fig. 1 Elemental feedback control system of fractional order $\alpha$

where $h$ is the time increment and $[x]$ means the integer part of $x$.

The "memory" effect of these operators is demonstrated by (1) and (2), where the convolution integral in (1) and the infinite series in (2), reveal the unlimited memory of these operators, ideal for modeling hereditary and memory properties in physical systems and materials.

An alternative definition to (1) and (2), which reveals useful for the analysis of fractional-order control systems, is given by the Laplace transform method. Considering vanishing initial conditions, the fractional differintegration is defined in the Laplace domain, $F(s)=L\{f(t)\}$, as:

$$
L\left\{a D_{t}^{\alpha} f(t)\right\}=s^{\alpha} F(s), \quad \alpha \in \Re .
$$

An important aspect of fractional-order algorithms can be illustrated through the elemental control system represented in Fig. 1, with open-loop transfer function $G(s)=K s^{-\alpha}(1<\alpha<2)$ in the forward path. The open-loop Bode diagrams of amplitude and phase have correspondingly a slope of $-20 \alpha \mathrm{dB} / \mathrm{dec}$ and a constant phase of $-\alpha \pi / 2$ rad over the entire frequency domain. Therefore, the closed-loop system has a constant phase margin of $\mathrm{PM}=\pi(1-\alpha / 2)$ rad that is independent of the system gain $K$. Likewise, this important property is also revealed through the root-locus depicted in Fig. $2(K \geq 0)$. In fact, when $1<\alpha<2$, the root-locus follows the relation $\pi-\pi / \pi \alpha=\cos ^{-1} \zeta$, where $\zeta$ is the damping ratio, independently of the gain $K$. Therefore, the closed-loop system will be robust against gain variations exhibiting step responses with an iso-damping property $[11,12]$.

\subsection{Approximations of fractional-order operators}

In this paper, we adopt discrete integer-order approximations to the fundamental element $s^{\alpha}(\alpha \in \mathrm{m})$ of a fractional-order control (FOC) strategy. The usual approach for obtaining discrete equivalents of continu- 


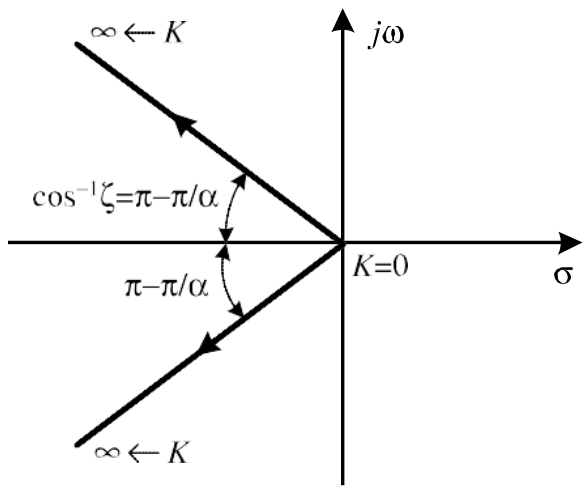

Fig. 2 Root-locus of $G(j \omega)$ for $1<\alpha<2, K \geq 0$

ous operators of type $s^{\alpha}$ adopts the Euler, Tustin, and Al-Alaoui generating functions [6-8].

It is well known that rational-type approximations

frequently converge faster than polynomial-type approximations and have a wider domain of convergence in the complex domain. Thus, by using the Euler operator $w\left(z^{-1}\right)=\left(1-z^{-1}\right) / T_{c}$, and performing a power series expansion of $\left[w\left(z^{-1}\right)\right]^{\alpha}=\left[\left(1-z^{-1}\right) / T_{c}\right]^{\alpha}$ gives the discretization formula corresponding to the Grünwald-Letnikov definition(2):

$$
\begin{aligned}
D^{\alpha}\left(z^{-1}\right) & =\left(\frac{1-z^{-1}}{T_{c}}\right)^{\alpha} \\
& =h^{\alpha}(0)+h^{\alpha}(1) z^{-1}+\cdots \\
& =\sum_{k=0}^{\infty} h^{\alpha}(k) z^{-k},
\end{aligned}
$$

where $T_{c}$ is the sampling period and $h^{\alpha}(k)$ is the impulse response sequence given by the expression $(k \geq 0)$ :

$$
h^{\alpha}(k)=\left(\frac{1}{T_{c}}\right)^{\alpha}\left(\begin{array}{c}
k-\alpha-1 \\
k
\end{array}\right) .
$$

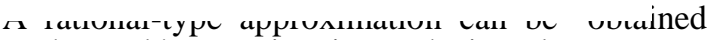
through a Padé approximation to the impulse response sequence (5) $h^{\alpha}(k)$, yielding the discrete transfer function:

$$
\begin{aligned}
H\left(z^{-1}\right) & =\frac{b_{0}+b_{1} z^{-1}+\cdots+b_{m} z^{-m}}{1+a_{1} z^{-1}+\cdots+a_{n} z^{-n}} \\
& =\sum_{k=0}^{\infty} h(k) z^{-k}
\end{aligned}
$$

where $m \leq n$ and the coefficients $a_{k}$ and $b_{k}$ are determined by fitting the first $m+n+1$ values of $h^{\alpha}(k)$ into the impulse response $h(k)$ of the desired approximation $H\left(z^{-1}\right)$. Thus, we obtain an approximation that has a perfect match to the desired impulse response $h^{\alpha}(k)$ for the first $m+n+1$ values of $k$ [8]. Note that the above Padé approximation is obtained by considering the Euler operator but the determination process will be exactly the same for other types of discretization schemes, such as the Tustin scheme.

\section{Heat diffusion}

The heat diffusion is governed by a linear partial differential equation (PDE) of the form:

$$
\frac{\partial u}{\partial t}=k\left(\frac{\partial^{2} u}{\partial x^{2}}+\frac{\partial^{2} u}{\partial y^{2}}+\frac{\partial^{2} u}{\partial z^{2}}\right),
$$

where $k$ is the diffusivity, $t$ is the time, $u$ is the temperature, and $(x, y, z)$ are the space Cartesian coordinates. The system (7) involves the solution of a PDE of parabolic type for which the standard theory guarantees the existence of a unique solution [13].

For the case of a planar perfectly isolated surface, we can apply a constant temperature $U_{0}$ at $x=0$, and we can analyze the heat diffusion along the horizontal coordinate $x$. Under these conditions, the heat diffusion phenomenon is described by a noninteger order model, yielding a transfer function of type:

$$
U(x, s)=\frac{U_{0}}{s} G(s), \quad G(s)=e^{-x \sqrt{\frac{s}{k}}}
$$

where $x$ is the space coordinate, $U_{0}$ is the boundary condition, and $G(s)$ is the system transfer function.

The solution of system (8) in the time domain yields

$$
\begin{aligned}
u(x, t) & =U_{0} \operatorname{erfc}\left(\frac{x}{2 \sqrt{k t}}\right) \\
& =U_{0}\left(1-\frac{2}{\sqrt{\pi}} \int_{0}^{\frac{x}{2 \sqrt{k t}}} e^{-u^{2}} d u\right) .
\end{aligned}
$$

In our study, the simulation of the heat diffusion is accomplished by adopting the Crank-Nicholson implicit numerical integration based on the discrete approximation to differentiation as [14]:

$$
-r u[j+1, i+1]+(2+r) u[j+1, i]
$$




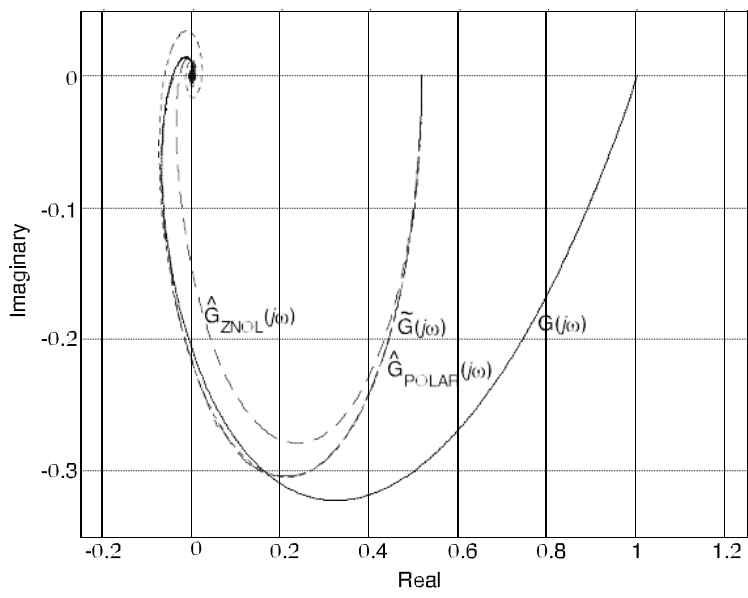

Fig. 3 Polar diagrams of $G(j \omega), \tilde{G}(j \omega), \hat{G}_{Z N O L}(j \omega)$, and $\hat{G}_{\text {Polar }}(j \omega)$, for $x=3.0 \mathrm{~m}$ and $k=0.042 \mathrm{~m}^{2} \mathrm{~s}^{-1}$

$$
\begin{aligned}
& -r u[j+1, i-1] \\
= & r u[j, i+1]+(2-r) u[j, i]+u[j, i-1],
\end{aligned}
$$

where $r=k L 1 t\left(L 1 x^{2}\right)^{-1},\{L 1 x, L 1 t\}$ and $\{i, j\}$ are the increments and the integration indices for space and time, respectively [13].

Figure 3 depicts the polar diagrams both for the theoretical $G(j \omega)$ (8) and numerical $\tilde{G}(j \omega)$ (i.e., from (8) and (10), respectively) implementations when $x=$ $3.0 \mathrm{~m}$ and $k=0.042 \mathrm{~m}^{2} \mathrm{~s}^{-1}$. It is verified [15] that the results obtained through the numerical approach differ somewhat from the analytical results in the low frequency range [16]. Equation (10) is adopted in the simulations, and, therefore, the smaller gain of $\tilde{G}(j \omega)$ will correspond to the introduction of extra losses at low frequencies.

\section{Control strategies for heat diffusion systems}

This section studies four strategies for the control of the heat diffusion system. Several tuning approaches and distinct control algorithms are compared. On one hand, are evaluated heuristics (ZNOL-Ziegler Nichols open loop) and optimization strategies (ISEintegral square error and ITSE — integral time square error). On the other hand, integer PID, fractional PID, and Smith Predictor (SP) control algorithms are considered.

In the Sects. 4.1 and 4.2, we analyze the system of Fig. 4 by adopting the classical integer-order
$\mathrm{PID}_{Z N O L}$ tuned through the $Z N O L$ heuristics and the fractional $\mathrm{PID}^{\beta}$, respectively. In the Sects. 4.3 and 4.4, we adopt a SP (Fig. 5) with a fractional $\mathrm{PID}^{\beta}$ controller $\left(\mathrm{SP}_{-} \mathrm{PID}{ }^{\beta}\right)$. In the first case, the approximation model $\hat{G}_{Z N O L}$, adopted in the SP, consists on the ZNOL scheme (algorithm denoted by SP_PID $\beta\left[\hat{G}_{Z N O L}\right]$ ). In the second case, the approximation model $\hat{G}_{\text {Polar }}$ results from minimization of square error between $\tilde{G}(j \omega)$ and $\hat{G}(j \omega)$ in the frequency domain (algorithm denoted by SP_PID $\left.{ }^{\beta}\left[\hat{G}_{\text {Polar }}\right]\right)$.

To conclude this section, in Sect. 4.5, we study the variation of the values of $\mathrm{PID}^{\beta}$ parameters when we change the characteristics of the diffusion system, namely, when we change the diffusivity constant $k$.

The effect of actuator saturation in the close loop system performance is also investigated for all cases.

The generalized PID controller $G_{c}(s)$ has a transfer function of the form [5]:

$$
G_{c}(s)=K\left[1+\frac{1}{T_{i} s^{\alpha}}+T_{d} s^{\beta}\right],
$$

where $\alpha$ and $\beta$ are the orders of the fractional integrator and differentiator, respectively. The constants $K$, $T_{i}$, and $T_{d}$ are correspondingly the proportional gain, the integral time constant, and the derivative time constant.

Clearly, taking $(\alpha, \beta)=\{(1,1),(1,0),(0,1),(0,0)\}$ we get the classical $\{$ PID, PI, PD, P $\}$ controllers, respectively. Other PID controllers are possible, namely: $\mathrm{PD}^{\beta}$ controller, $\mathrm{PI}^{\alpha}$ controller, $\mathrm{PID}^{\beta}$ controller, and so on. The $\mathrm{PI}^{\alpha} \mathrm{D}^{\beta}$ controller is more flexible and gives the possibility of adjusting more carefully the closed-loop system characteristics [17].

\subsection{The PIDZNOL}

In this subsection, we analyze the closed-loop system with a conventional PID controller given by the transfer function (11) with $\alpha=\beta=1$. Often, the PID parameters $\left(K, T_{i}, T_{d}\right)$ are tuned by using the so called Ziegler Nichols open loop (ZNOL) method [18]. The $Z N O L$ heuristics are based on the approximate firstorder plus dead-time model:

$$
\hat{G}_{\mathrm{ZNOL}}(s)=\frac{K_{p}}{\tau s+1} e^{-s T} .
$$

For the heat system the model parameters are $\left\{K_{p}, \tau, T\right\}=\{0.52,162,28\}$ leading to the PID constants $\left\{K, T_{i}, T_{d}\right\}=\{18.07,34.0,8.5\}$. 
Fig. 4 Closed-loop system with PID controller $G_{c}(s)$
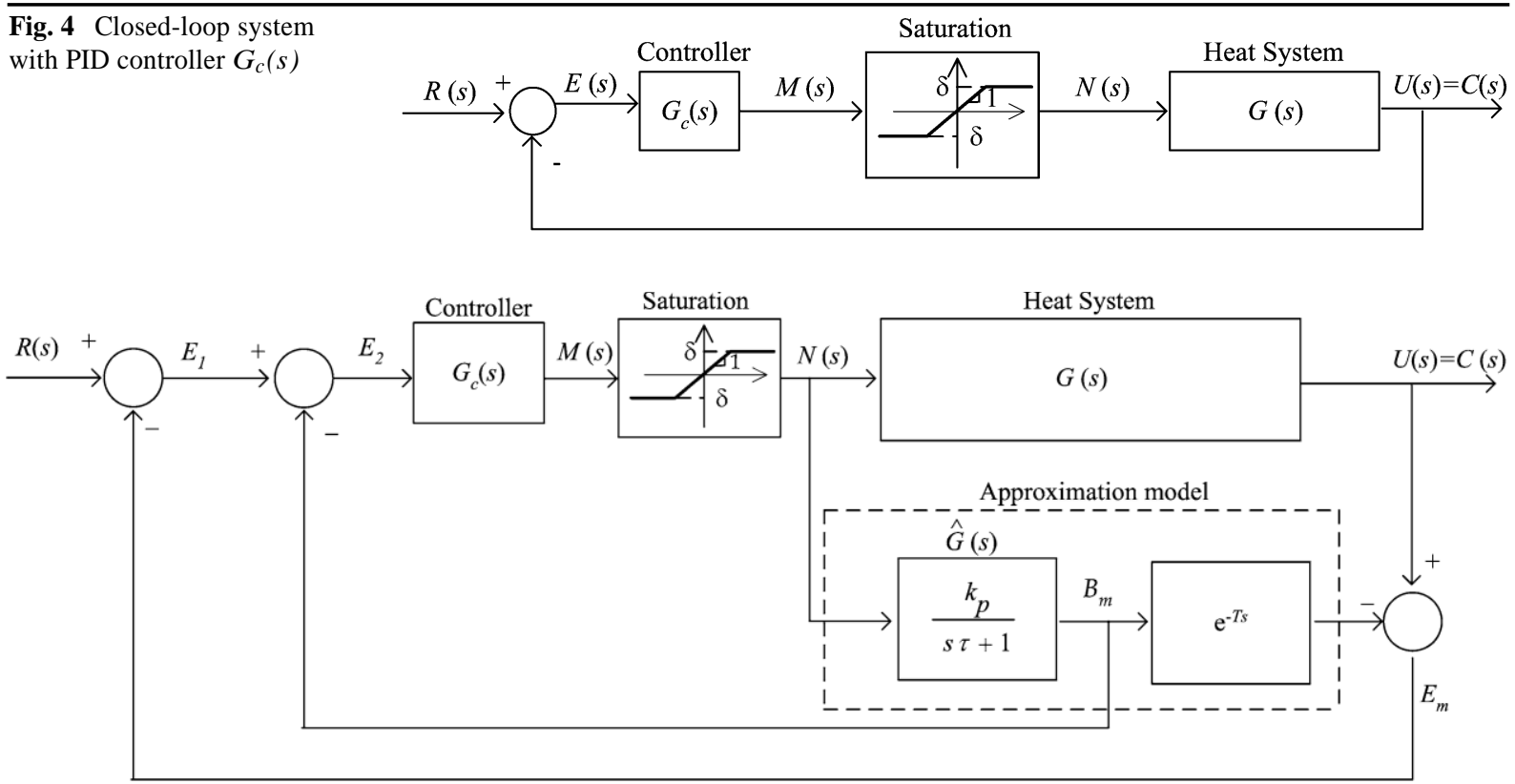

Fig. 5 Closed-loop system SP_PID ${ }^{\beta}$ of the Smith predictor with a fractional $\mathrm{PID}^{\beta}$ controller $G_{C}(s)$

A step input $r(t)$ is applied at $x=0.0 \mathrm{~m}$ and the output $c(t)$ analyzed for $x=3.0 \mathrm{~m}$ (Fig. 6), without actuator saturation $(\delta=\infty)$.

We verify that the system with a $\mathrm{PID}_{Z N O L}$ does not produce satisfactory results leading to a significant overshoot $o v$, a large settling time $t_{s}$ and a time delay $t_{d}$. In fact, we get $\left\{t_{s}, t_{r}, t_{p}, o v(\%), t_{d}\right\} \equiv$ $\{44.8,12.0,27.5,68.56 \%, 3.0\}$, where $t_{p}$ represents the peak time and $t_{r}$ the rise time.

We analyze two indices that measure the response error, namely, the ISE and the ITSE criteria defined as:

$$
\begin{aligned}
& \text { ISE }=\int_{0}^{\infty}[r(t)-c(t)]^{2} d t, \\
& \text { ITSE }=\int_{0}^{\infty} t[r(t)-c(t)]^{2} d t .
\end{aligned}
$$

We can use other integral performance criteria such as the integral absolute error (IAE) or the integral time absolute error (ITAE). In the present case, the ISE and the ITSE criteria have produced the best results and are adopted in the sequel. Furthermore, the ITSE criterion enable us to study the influence of time in the error generated by the system.
Another possible performance index consists on the energy $E_{m}$ at the PID controller output $m(t)$ given by the expression:

$$
E_{m}=\int_{0}^{T_{\varepsilon}} m^{2}(t) d t,
$$

where $T_{e}$ is a time window sufficiently large to stabilize the systems output $c(t)$ at the steady state. In this case, the PID reveals the following values for parameters (ISE, ITSE, $\left.E_{m}\right)=\left(27.53,613.97,2.52 \times 10^{5}\right)$ when adopting $T_{e}=700 \mathrm{~s}$.

The step response of Fig. 6 reveals a large time delay and a considerable overshoot. The poor results obtained indicate that the method of tuning as well the structure of the system may not be the most adequate for the control of the heat system under consideration.

In this perspective, we propose the use of fractionalorder controllers tuned by the minimization of the indices ISE and ITSE and the SP structure to achieve a superior control of this type of systems.

4.2 The $\mathrm{PID}^{\beta}$ : Controller tuning using the optimization indices ISE and ITSE

In this section, we analyze the closed-loop system with a fractional $\mathrm{PI}^{\alpha} \mathrm{D}^{\beta}$ controller given by the transfer 


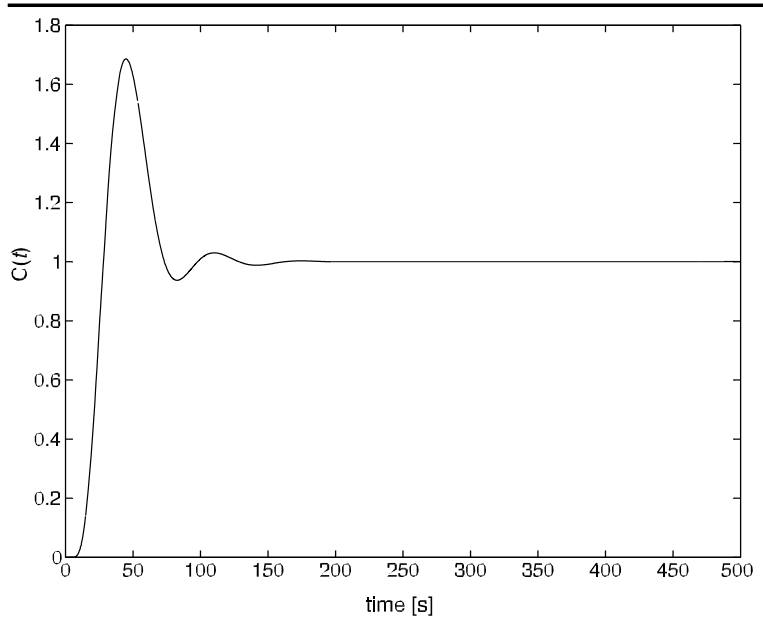

Fig. 6 Step responses of the closed-loop system for the PID controller and $x=3.0 \mathrm{~m}, k=0.042 \mathrm{~m}^{2} \mathrm{~s}^{-1}$

function (11) with $\alpha=1$. The fractional-order derivative term $T_{d} \mathrm{~s}^{\beta}$ in (11) is implemented by using a 4th-order Padé discrete rational transfer function of type (6). It used a sampling period of $T_{C}=0.1 \mathrm{~s}$. The $\mathrm{PID}^{\beta}$ controller is tuned by minimization the ISE (13) or, alternatively, the ITSE (14) criteria.

A step reference input $R(s)=1 / s$ is applied at $x=$ $0.0 \mathrm{~m}$ and the output $c(t)$ is analyzed for $x=3.0 \mathrm{~m}$ without actuator saturation. The heat system is simulated for 3,000 seconds and is considered $T_{e}=700 \mathrm{~s}$. Figure 7 illustrates the variation of the fractional PID parameters $\left(K, T_{i}, T_{d}\right)$ as a function of the order's derivative $\beta$ when minimizing the ISE and the ITSE criteria. The dots represent the values corresponding to the classical PID ZNOL $_{\text {addressed in the previous sec- }}$ tion.

The curves reveal that for $\beta<0.4$, the parameters $\left(K, T_{i}, T_{d}\right)$ are slightly different for the ISE and ITSE criteria. However, for $\beta \geq 0.4$, they lead almost to similar values. This fact indicates a large influence of a weak order derivative on system's dynamics.

To further illustrate the performance of the $\operatorname{PID}^{\beta}$ a saturation nonlinearity is included in the closed-loop system of Fig. 4 and inserted in series with the output of the PID controller $G_{c}(s)$. The saturation element is defined as:

$$
n(m)= \begin{cases}m, & |m|<\delta, \\ \delta \operatorname{sign}(m), & |m| \geq \delta .\end{cases}
$$

The controller performance is evaluated for different values of $\delta$, namely, for $\delta=\{40,60,80,100, \infty\}$, where the last value corresponds to a system without saturation. In the simulations, we use the same fractional-PID parameters obtained without considering the saturation nonlinearity.

Figures 8 and 9 show the step responses of the closed-loop system and the corresponding controller output for the $\mathrm{PID}^{\beta}$ tuned in the ISE and ITSE perspectives and for $\delta=40$ and $\delta=\infty$, respectively. The controller parameters, corresponding to the minimization of those indices, lead to the values ISE: $\left\{K, T_{i}, T_{d}, \beta\right\} \equiv\{3,23,90.6,0.875\}$ and ITSE: $\left\{K, T_{i}, T_{d}, \beta\right\} \equiv\{1.8,17.6,103.6,0.85\}$.

The step responses of the $\mathrm{PID}^{\beta}$ reveal a large diminishing of the overshoot and the rise time when compared with the integer PIDZNOL, showing a good transient response and a zero steady-state error. These results demonstrate the effectiveness of the fractional algorithms when used for the control of fractionalorder systems. Furthermore, the step response and the controller output are also improved when actuator saturation occurs.

Figures 10 and 11 show the variation of $t_{s}, t_{r}, t_{p}$ and $o v(\%)$ versus $\beta$, for the closed-loop response tuned through the minimization of the ISE and the ITSE indices, respectively.

The charts reveal several different regions. Furthermore, it is clear that it is impossible to simultaneously minimize all parameters. However, for $\beta_{\text {ISE }} \approx 0.875$ and $\beta_{\text {ITSE }} \approx 0.85$, we get a good compromise between all possibilities.

The energy $E_{m}(15)$ at the output $m(t)$ of the $\mathrm{PID}^{\beta}$ controller is also analyzed. Figure 12 depicts the energy $E_{m}$ as function of the ISE and the ITSE indices, for $0 \leq \beta \leq 1$. As can be seen, the energy changes smoothly for different values of $\delta$ when considering a given order $\beta$.

However, fixing the value $\delta$, we verify that the energy $E_{m}$ increases significantly with $\beta$.

On the other hand, we observe that the ISE decreases with $\delta$ for $\beta \leq 0.875$, while for $\beta>0.875$ the ISE increases very quickly. The same conclusions can be. gntthinedrelatively to the eTTSE griterion. but for $\beta=$

the system particularly for low values of the fractionalorder derivative term.

When comparing the two indices, we also verify that the values for the ITSE are generally larger than those for the ISE. This occurs due to the large simulation time needed to stabilize the system, which is about $T_{e} \sim 700 \mathrm{~s}$. 

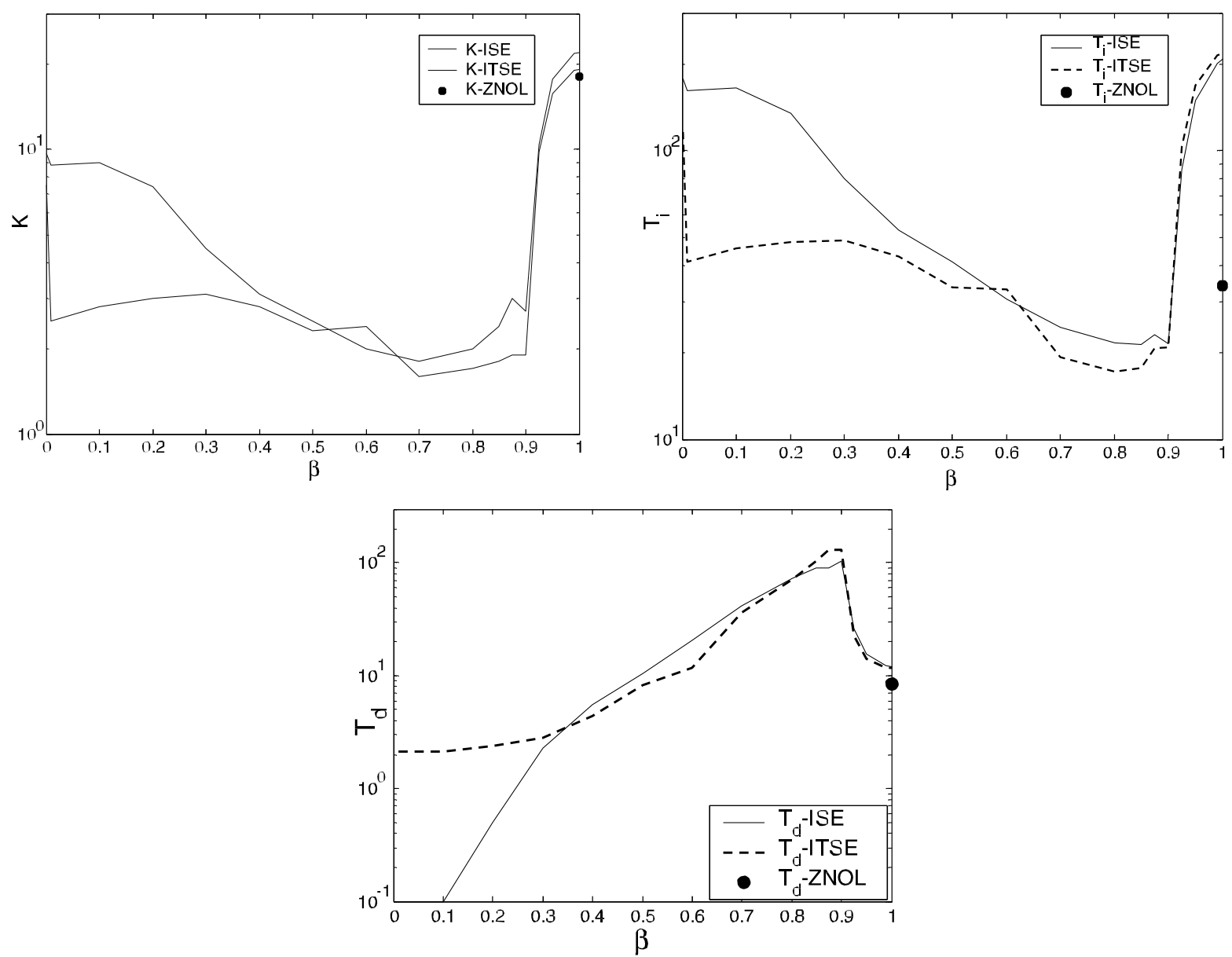

Fig. 7 The $\operatorname{PID}^{\beta}$ parameters $\left(K, T_{i}, T_{d}\right)$ versus $\beta$ for the ISE and the ITSE criteria $(\delta=\infty), k=0.042 \mathrm{~m}^{2} \mathrm{~s}^{-1}$. The $d o t$ represents the PID $Z$ ZNOL
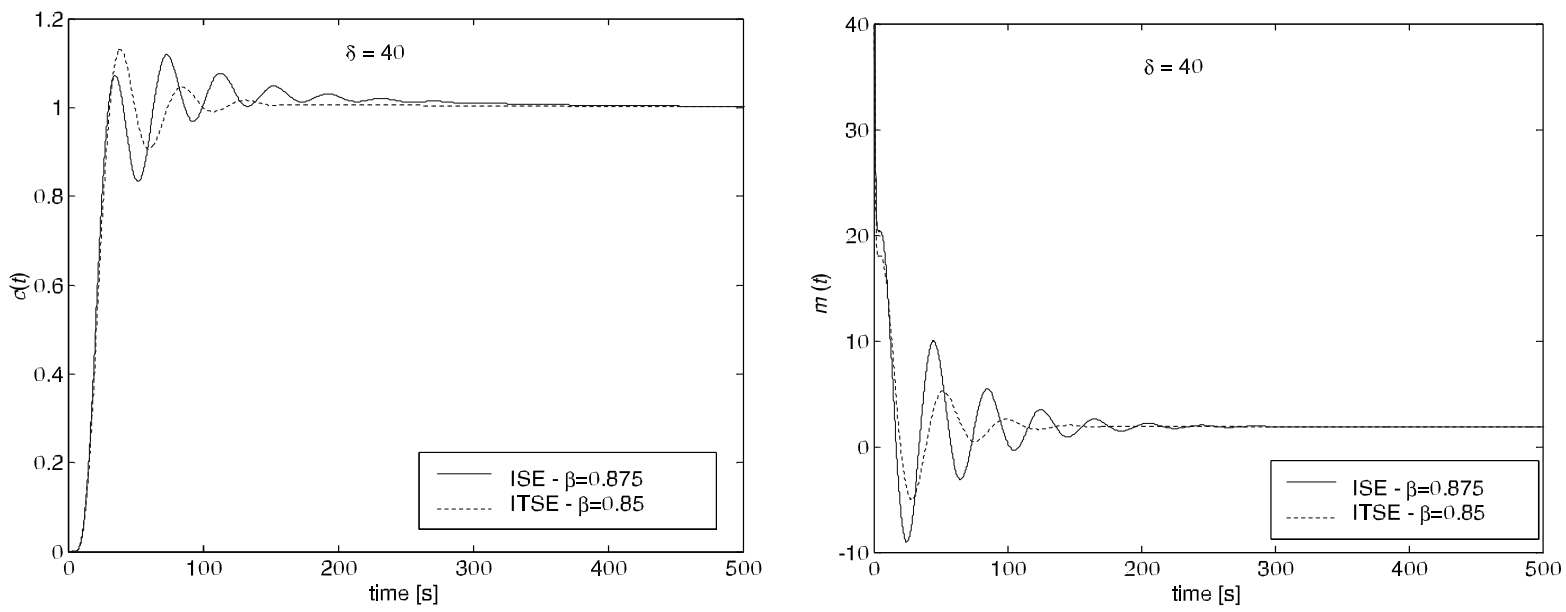

Fig. 8 Step responses of the closed-loop system and the controller output for the ISE and the ITSE indices, with a PID ${ }^{\beta}$ controller, $\delta=40, x=3.0 \mathrm{~m}, k=0.042 \mathrm{~m}^{2} \mathrm{~s}^{-1}$ 

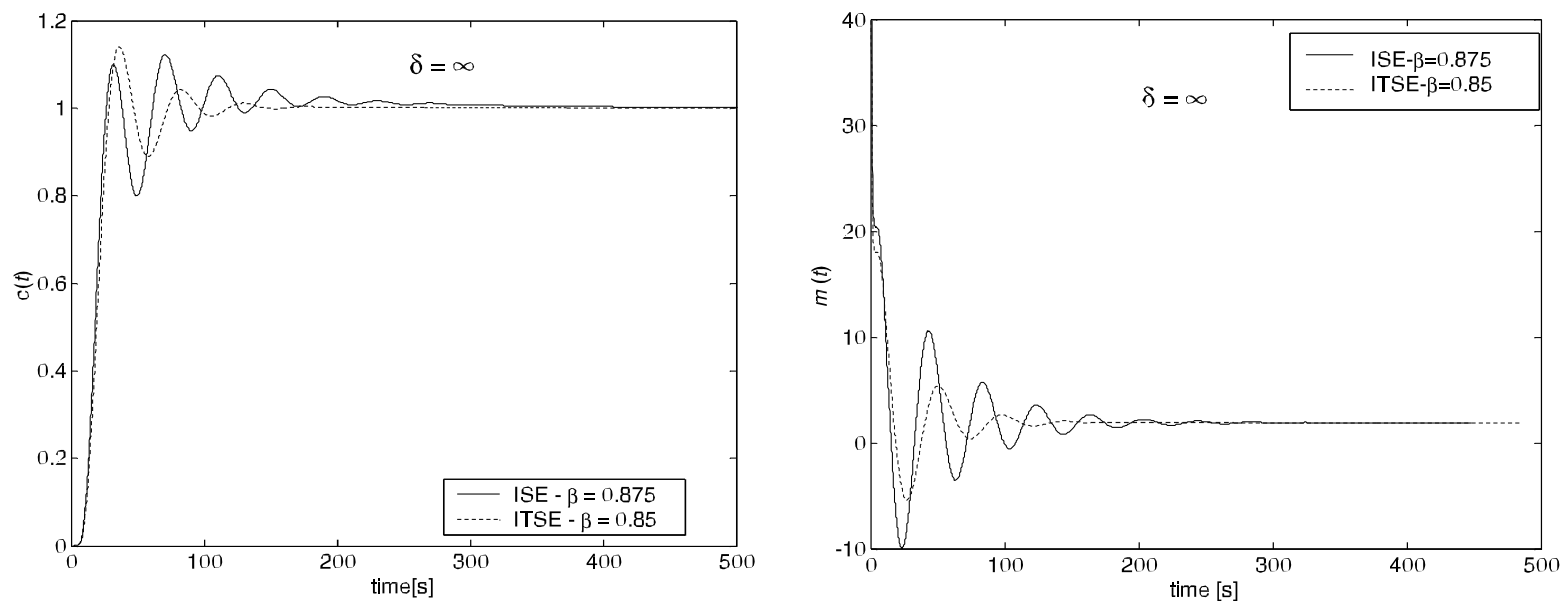

Fig. 9 Step responses of the closed-loop system and the controller output for the ISE and the ITSE indices, with a PID ${ }^{\beta}$ controller, $\delta=\infty, x=3.0 \mathrm{~m}, k=0.042 \mathrm{~m}^{2} \mathrm{~s}^{-1}$
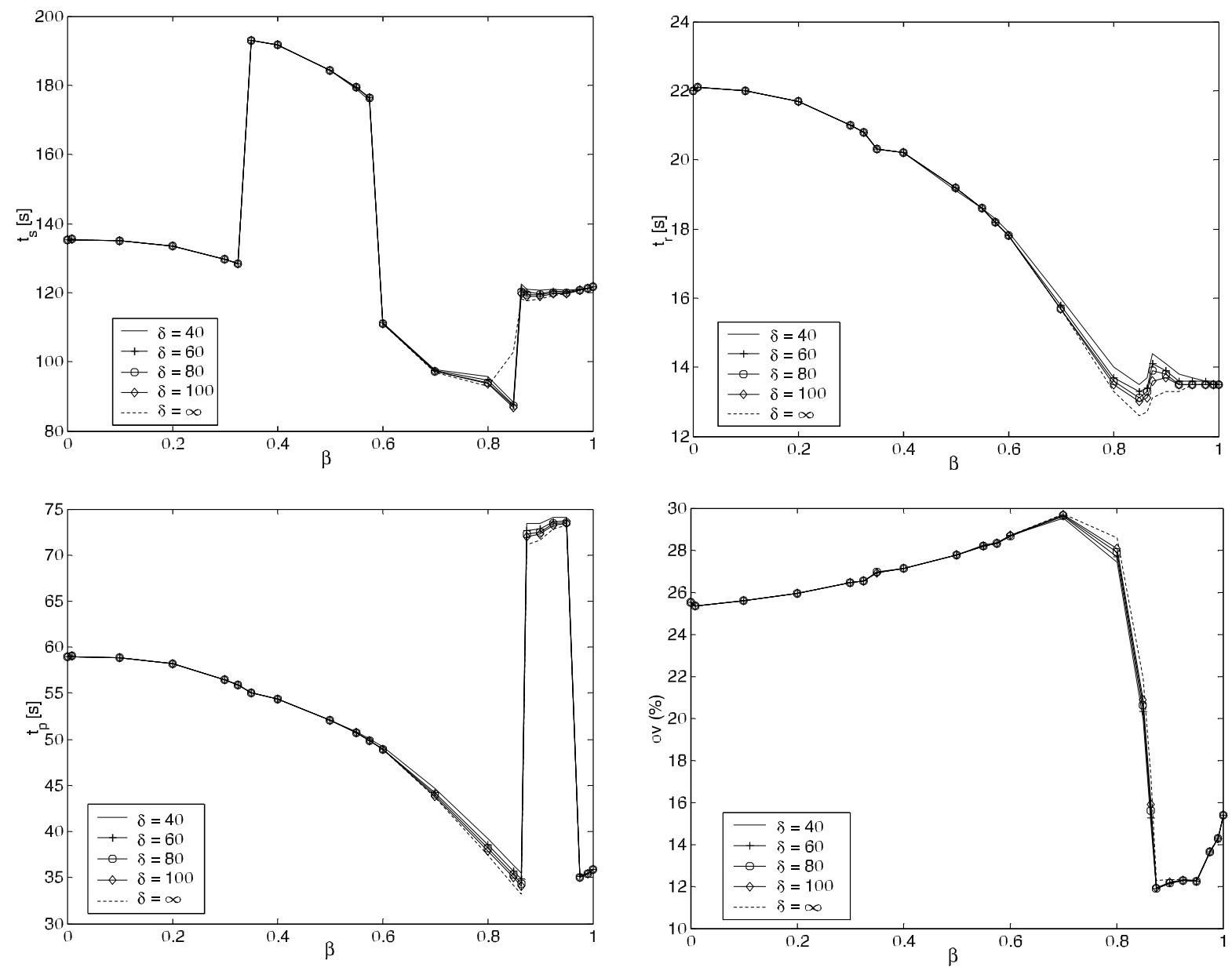

Fig. 10 Parameters $t_{s}, t_{r}, t_{p}, o v(\%)$ versus $\beta$ for the step responses of the closed-loop system for the ISE, with a PID $\beta$ controller, when $\delta=\{40,60,80,100, \infty\}, x=3.0 \mathrm{~m}, k=0.042 \mathrm{~m}^{2} \mathrm{~s}^{-1}$ 

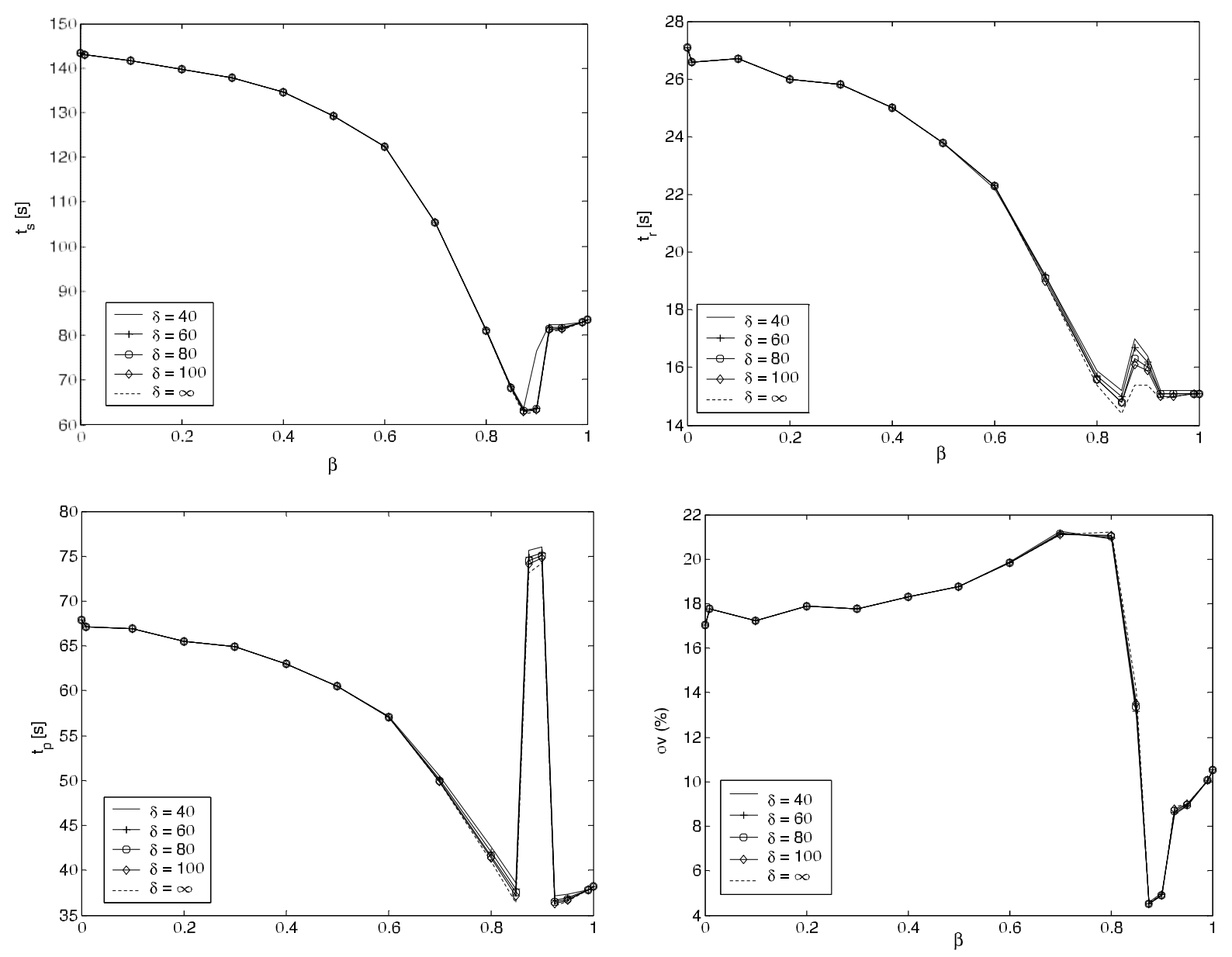

Fig. 11 Parameters $t_{s}, t_{r}, t_{p}, o v(\%)$ versus $\beta$ for the step responses of the closed-loop system for the ITSE, with a PID ${ }^{\beta}$ controller, when $\delta=\{40,60,80,100, \infty\}, x=3.0 \mathrm{~m}, k=0.042 \mathrm{~m}^{2} \mathrm{~s}^{-1}$
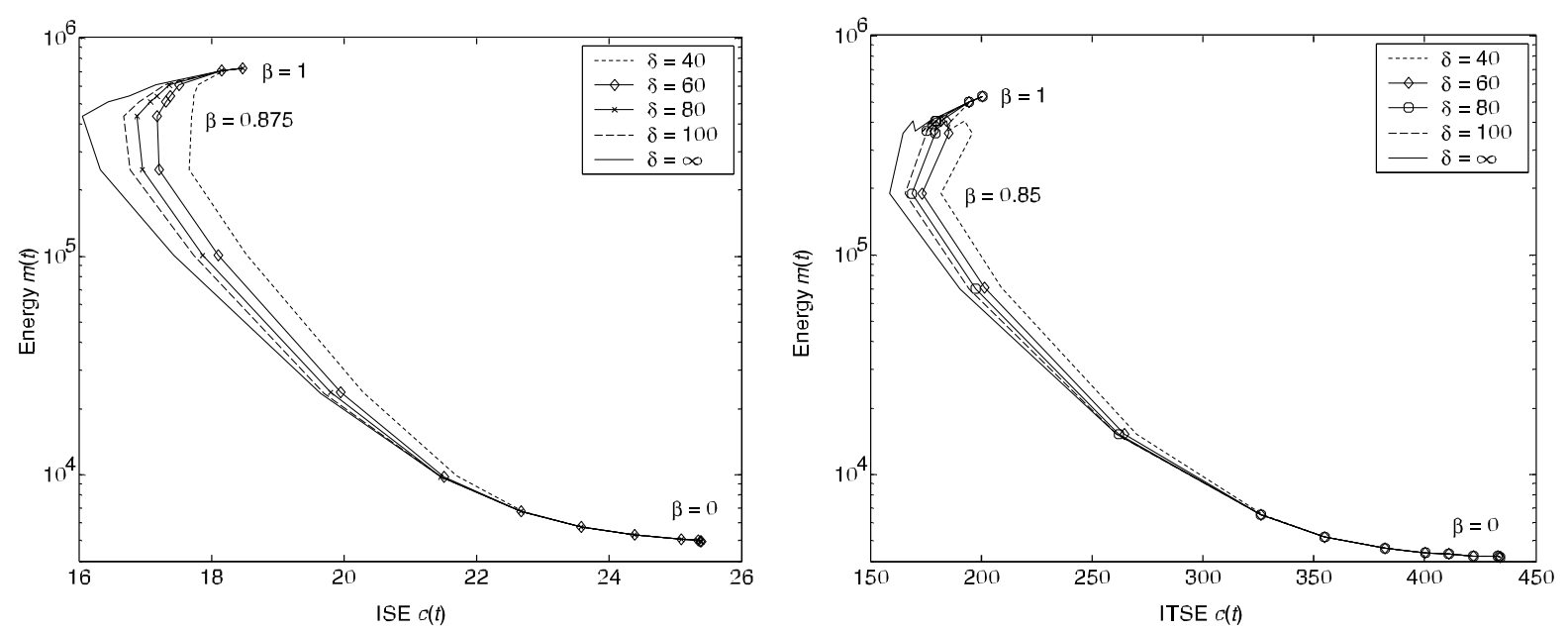

Fig. 12 Energy $E_{m}$ versus the ISE and the ITSE for $\delta=\{40,60,80,100, \infty\}, 0 \leq \beta \leq 1, k=0.042 \mathrm{~m}^{2} \mathrm{~s}^{-1}$ 

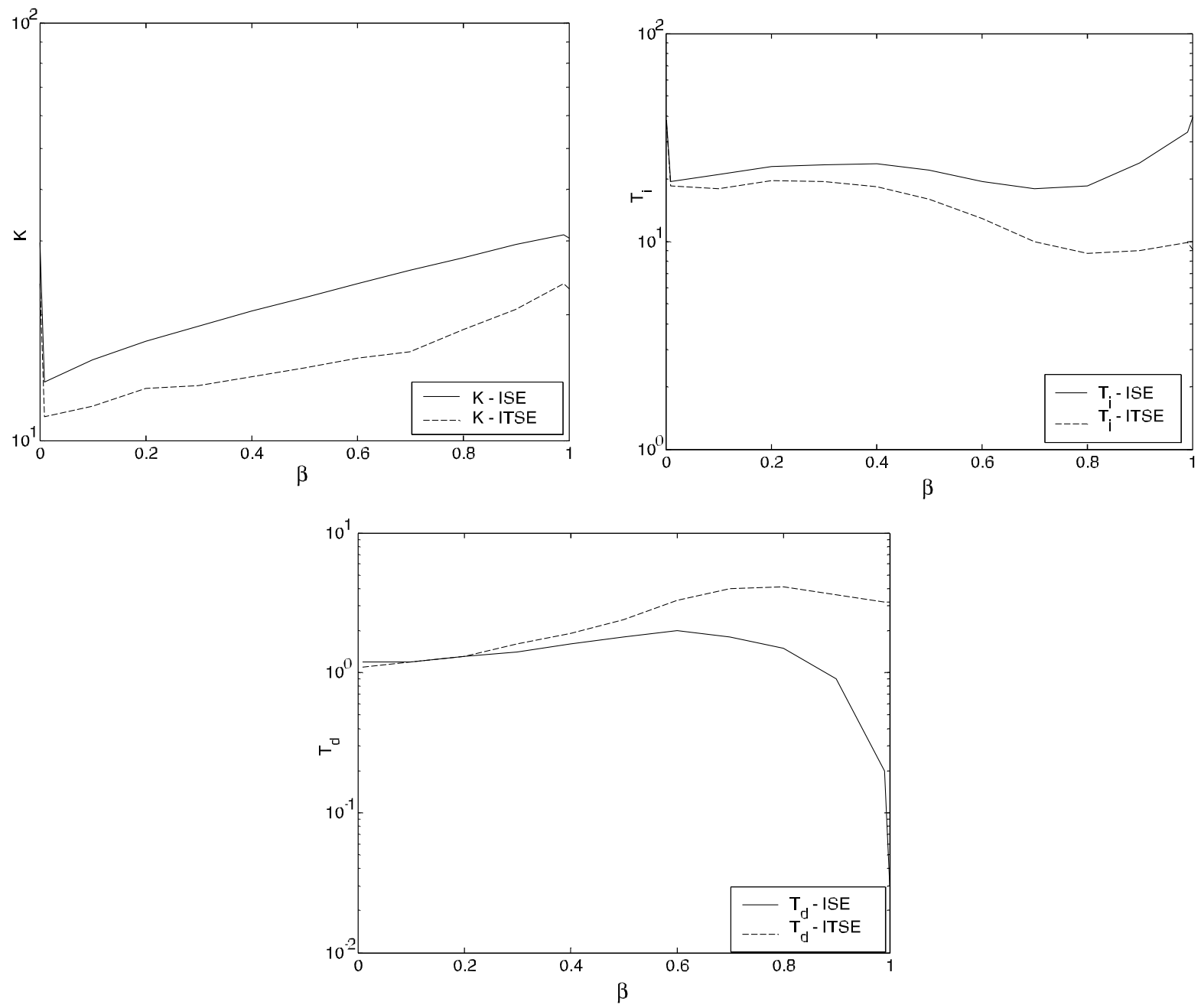

Fig. 13 The SP_PID $\beta$ parameters $\left(K, T_{i}, T_{d}\right)$ versus $\beta$ for the ISE and the ITSE criteria $(\delta=\infty), k=0.042 \mathrm{~m}^{2} \mathrm{~s}^{-1}$

In conclusion, for $0.85 \leq \beta \leq 0.875$, we get the best controller tuning, superior to the performance revealed by the classical integer-order scheme. The step responses reveal a large diminishing of the overshoot and the rise time when compared with the integer PID, showing a good transient response and a zero steadystate error. These results demonstrate the effectiveness of the fractional-order algorithms when used for the control of fractional-order systems.

\subsection{The SP_PID ${ }^{\beta}\left[\hat{G}_{Z N O L}\right]:$ Controller tuning using the optimization indices ISE and ITSE}

In this subsection, we adopt a fractional PID $^{\beta}$ controller inserted in a SP as represented in Fig. 5
This algorithm constitutes a dead-time compensation technique, very effective in improving the control of processes having time delays $[18,19]$.

The transfer function $\hat{G}(s)$, inserted in the second branch of the SP, consists in an approximation of a first-order plus dead-time model. For the Ziegler Nichols open loop heuristics, we get:

$$
\hat{G}_{\mathrm{ZNOL}}(s)=\frac{0.52}{162 s+1} e^{-28 s} .
$$

The SP_PID ${ }^{\beta}$ is tuned by applying the ISE and the ITSE criteria and its performance is evaluated for $\delta=\{40,60,80,100, \infty\}$. In the simulations including saturation, we maintain the fractional-PID parameters 

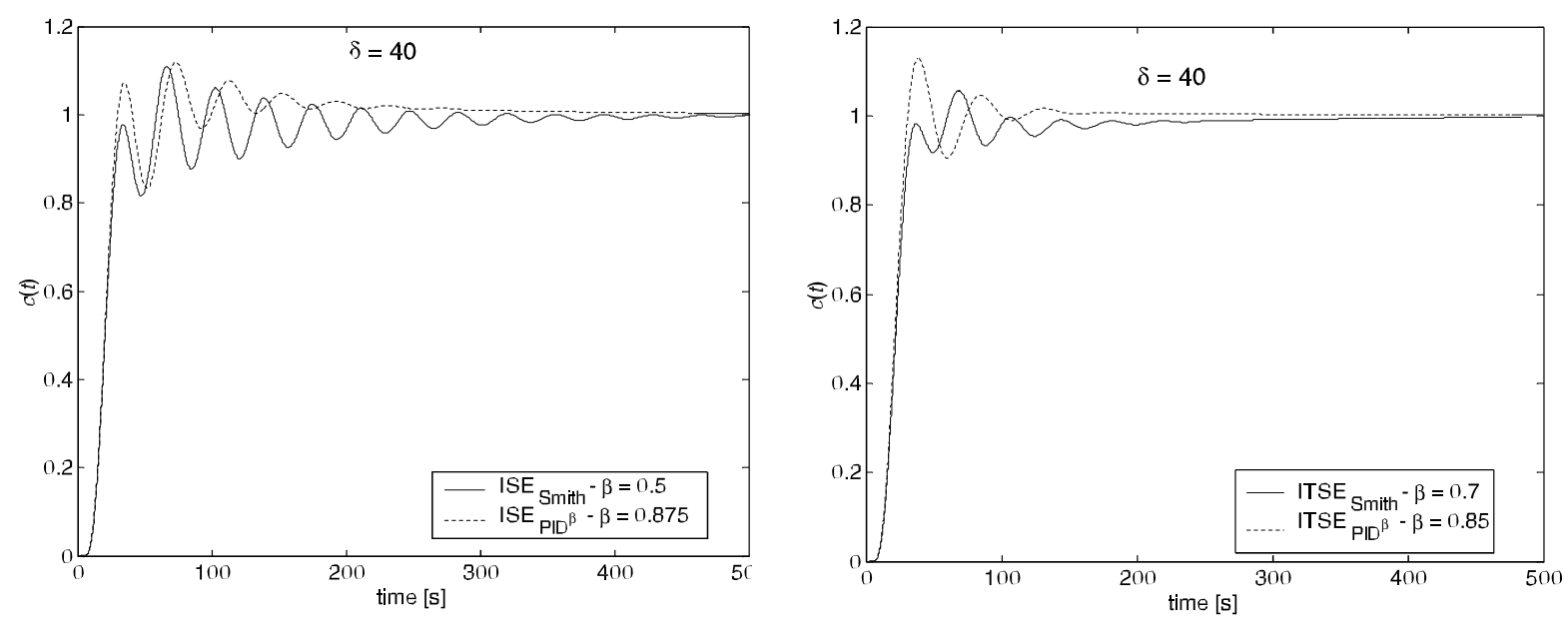

Fig. 14 Step responses of the closed-loop system for the PID $^{\beta}$ and the SP_PID ${ }^{\beta}$, for the ISE and the ITSE indices, with the optimal values of $\beta, \delta=40, x=3.0 \mathrm{~m}, k=0.042 \mathrm{~m}^{2} \mathrm{~s}^{-1}$
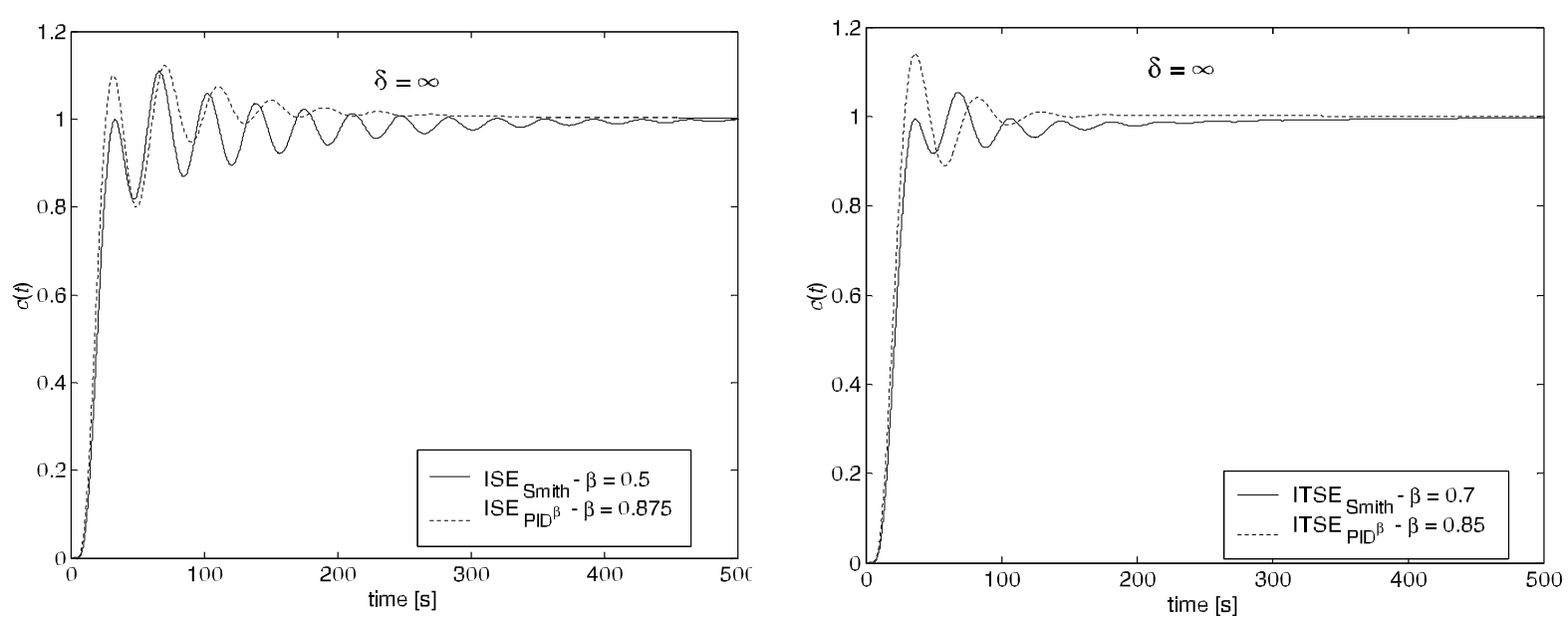

Fig. 15 Step responses of the closed-loop system for the PID $^{\beta}$ and the SP_PID ${ }^{\beta}$, for the ISE and the ITSE indices, with the optimal values of $\beta, \delta=\infty, x=3.0 \mathrm{~m}, k=0.042 \mathrm{~m}^{2} \mathrm{~s}^{-1}$

obtained previously, that is, without considering the saturation nonlinearity.

Figure 13 illustrates the variation of the SP-PID ${ }^{\beta}$ parameters $\left(K, T_{i}, T_{d}\right)$ as function of the order's derivative $\beta$, for the ISE and the ITSE criteria, without actuator saturation.

Figures 14 and 15 illustrate the step responses of the closed-loop system for $x=3.0 \mathrm{~m}$, when applying a unit step input $R(s)=1 / s$ at $x=0.0 \mathrm{~m}$, for the SP_PID $\beta, \delta=40$ and $\delta=\infty$. Both for the ISE and the ITSE, we depict the response corresponding to the best value of $\beta$, namely, $\left(\beta_{\text {ISE }}\right.$, $\left.\beta_{\text {ITSE }}\right)=(0.5,0.7)$ for the SP_PID ${ }^{\beta}$, and $\left(\beta_{\text {ISE }}\right.$, $\left.\beta_{\text {ITSE }}\right)=(0.875,0.85)$ for the simple $\operatorname{PID}^{\beta}$, respectively.

In these figures, we verify that the $\mathrm{PID}^{\beta}$ controller presents better results for the transient responses than those obtained for the SP_PID ${ }^{\beta}$, namely, a smaller $t_{s}$, and similar $t_{d}$ and $t_{r}$; however, the percentile overshoot $o v(\%)$ is smaller for the SP_PID ${ }^{\beta}$ controller.

Figures 16 and 17 depict the variation of the transient response parameters, $t_{s}, t_{r}, t_{p}$ and $o v(\%)$ versus $\beta$, for the SP_PID ${ }^{\beta}\left[\hat{G}_{Z N O L}\right]$ tuned through the minimization of the ISE and the ITSE indices, respectively. 

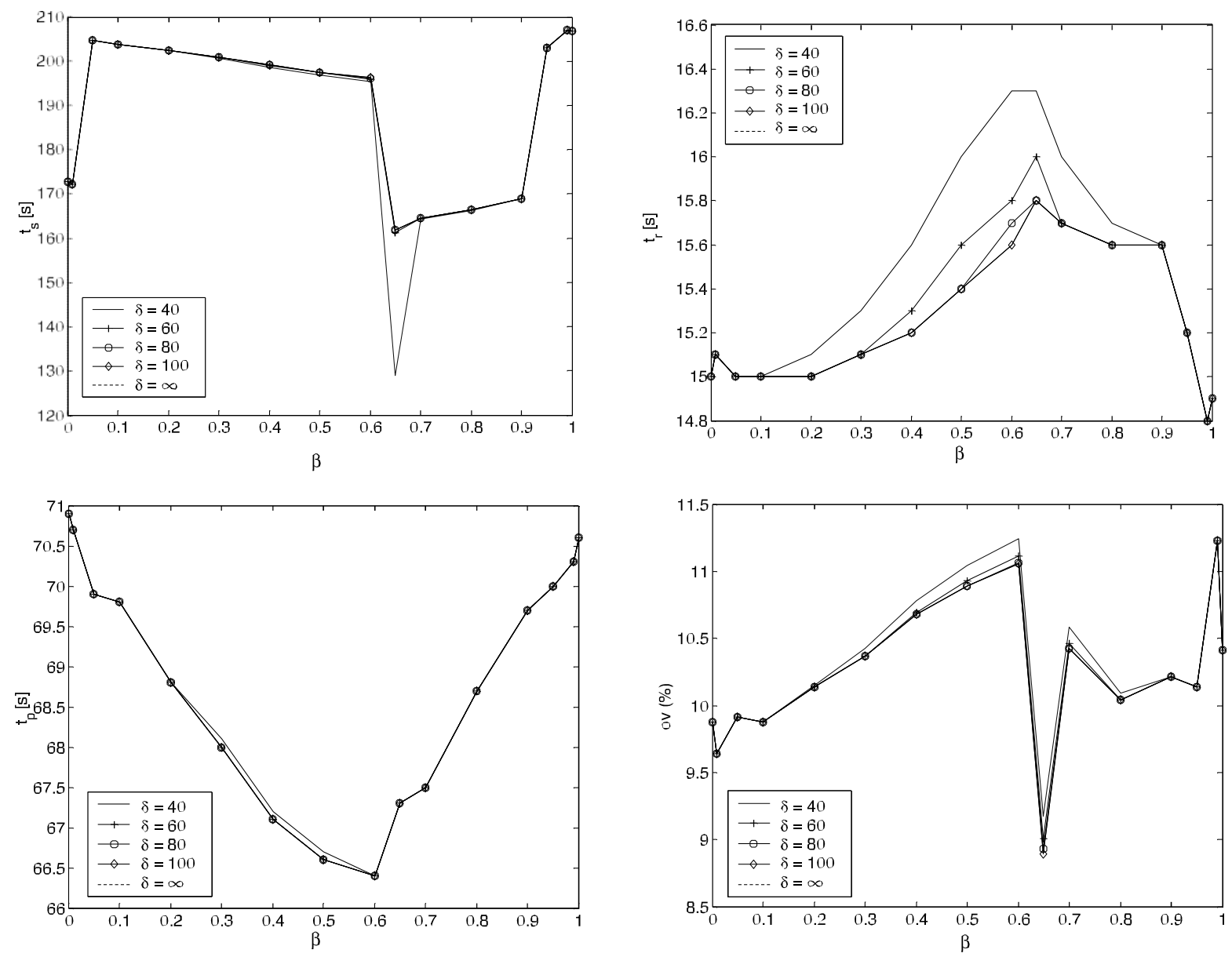

Fig. 16 Parameters $t_{s}, t_{r}, t_{p}$, ov $(\%)$ for the step responses of the closed-loop system with a SP_PID ${ }^{\beta}\left[\hat{G}_{Z N O L}\right]$, for the ISE, $\delta=\{40,60,80,100, \infty\}$ and $x=3.0 \mathrm{~m}, k=0.042 \mathrm{~m}^{2} \mathrm{~s}^{-1}$

Again, the charts reveal several distinct regions. The best compromise situations are $\beta_{\text {ISE }} \approx 0.5$ and $\beta_{\text {ITSE }} \approx 0.7$.

Both for the ISE and for ITSE indices, the parameters $t_{s}, t_{r}, t_{p}$, and $o v(\%)$ have the worst values for the case of $\beta$ being of integer order that lead us to confirm the benefits of the use of fractional order concepts.

Figure 18 depicts the relation between the controller action energy $E_{m}$ and the ISE and ITSE indices. Once again, we verify that the best case is achieved when $\left(\beta_{\text {ISE }}, \beta_{\text {ITSE }}\right)=(0.5,0.7)$.

Based on these results, we conclude that with the $\mathrm{PID}^{\beta}$ we can find betters results than the those obtained with the SP_PID ${ }^{\beta}\left[\hat{G}_{Z N O L}\right]$. Furthermore, the $\mathrm{PID}^{\beta}$ is advantageous because its implementation is considerably easier than the SP algorithm.
The time delay, observed in the Smith predictor step response, revealed an insufficient match between the system model $\tilde{G}(j \omega)$ and the first-order approximation $\hat{G}_{Z N O L}(j \omega)$.

In this line of thought, we decided to repeat the study of the SP with an other first-order approximation model, resulting from an approximation between the system model $\tilde{G}(j \omega)$ and $\hat{G}(j \omega)$ in the frequency domain (Fig. 3).

\subsection{The SP_PID ${ }^{\beta}\left[\hat{G}_{\text {Polar }}\right]$ : Controller tuning using the optimization indices ISE and ITSE}

In this section, the approximation model $\hat{G}(s)$, inserted in the second branch of the SP, is described by a first-order plus dead-time model with parameters esti- 

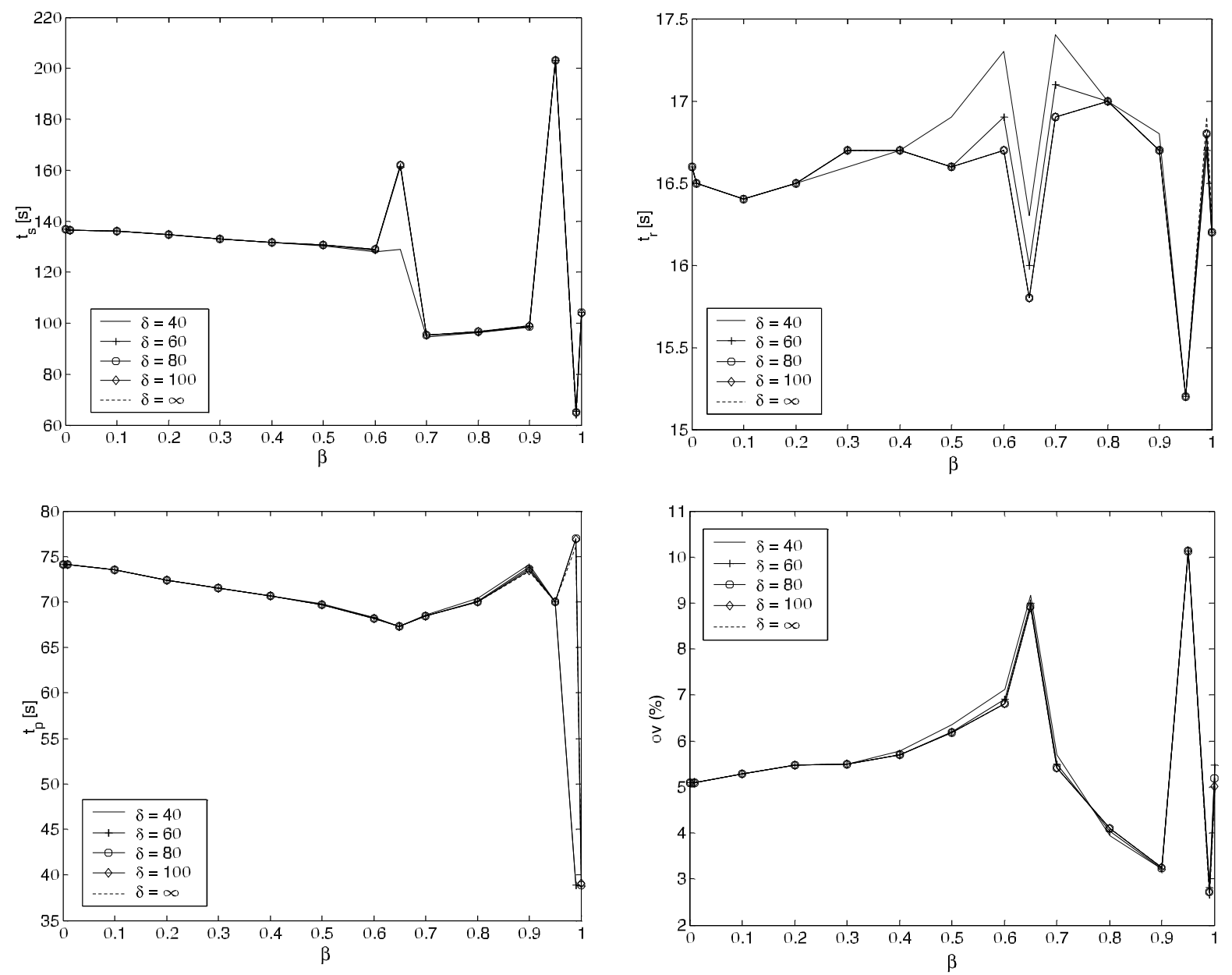

Fig. 17 Parameters $t_{s}, t_{r}, t_{p}$, ov (\%) for the step responses of the closed-loop system with a SP_PID $\beta\left[\hat{G}_{Z N O L}\right]$, for the ITSE, $\delta=\{40,60,80,100, \infty\}$ and $x=3.0 \mathrm{~m}, k=0.042 \mathrm{~m}^{2} \mathrm{~s}^{-1}$
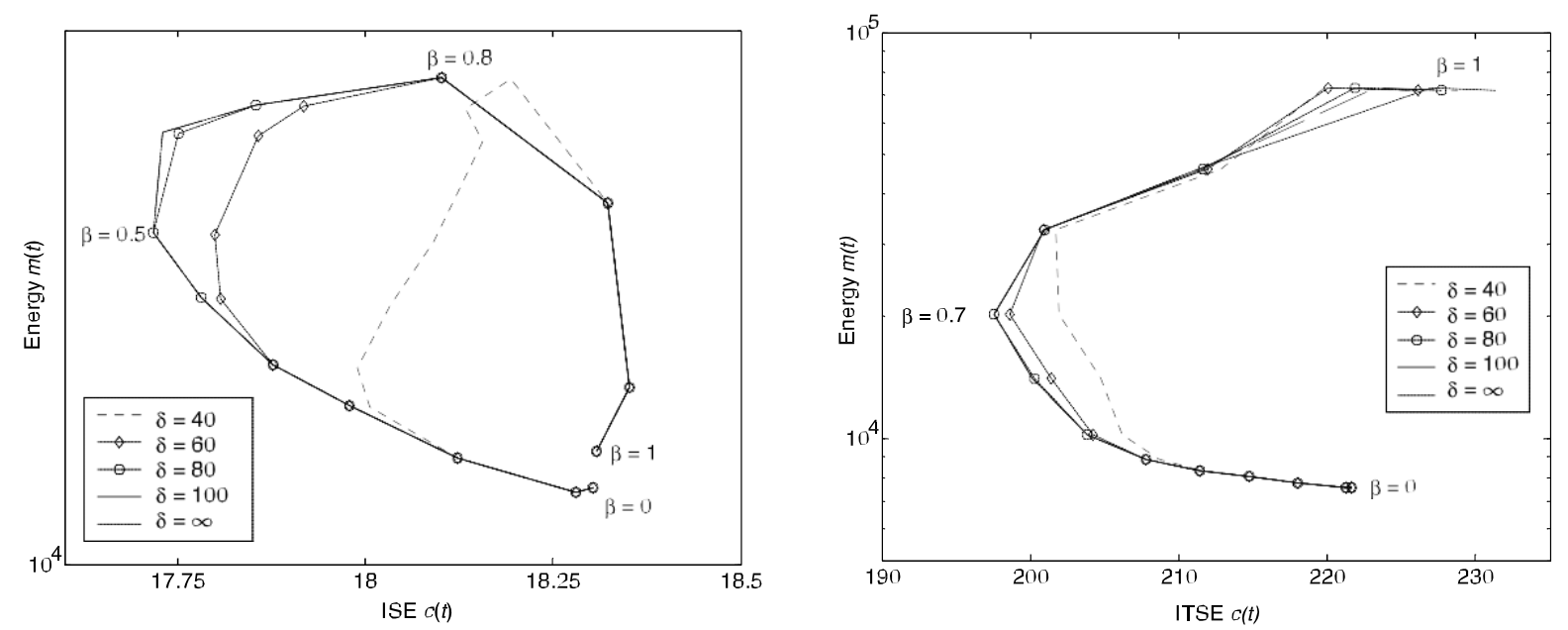

Fig. 18 Energy $E_{m}$ versus the ISE and the ITSE indices for $\delta=\{40,60,80,100, \infty\}, 0 \leq \beta \leq 1, k=0.042 \mathrm{~m}^{2} \mathrm{~s}^{-1}$ 

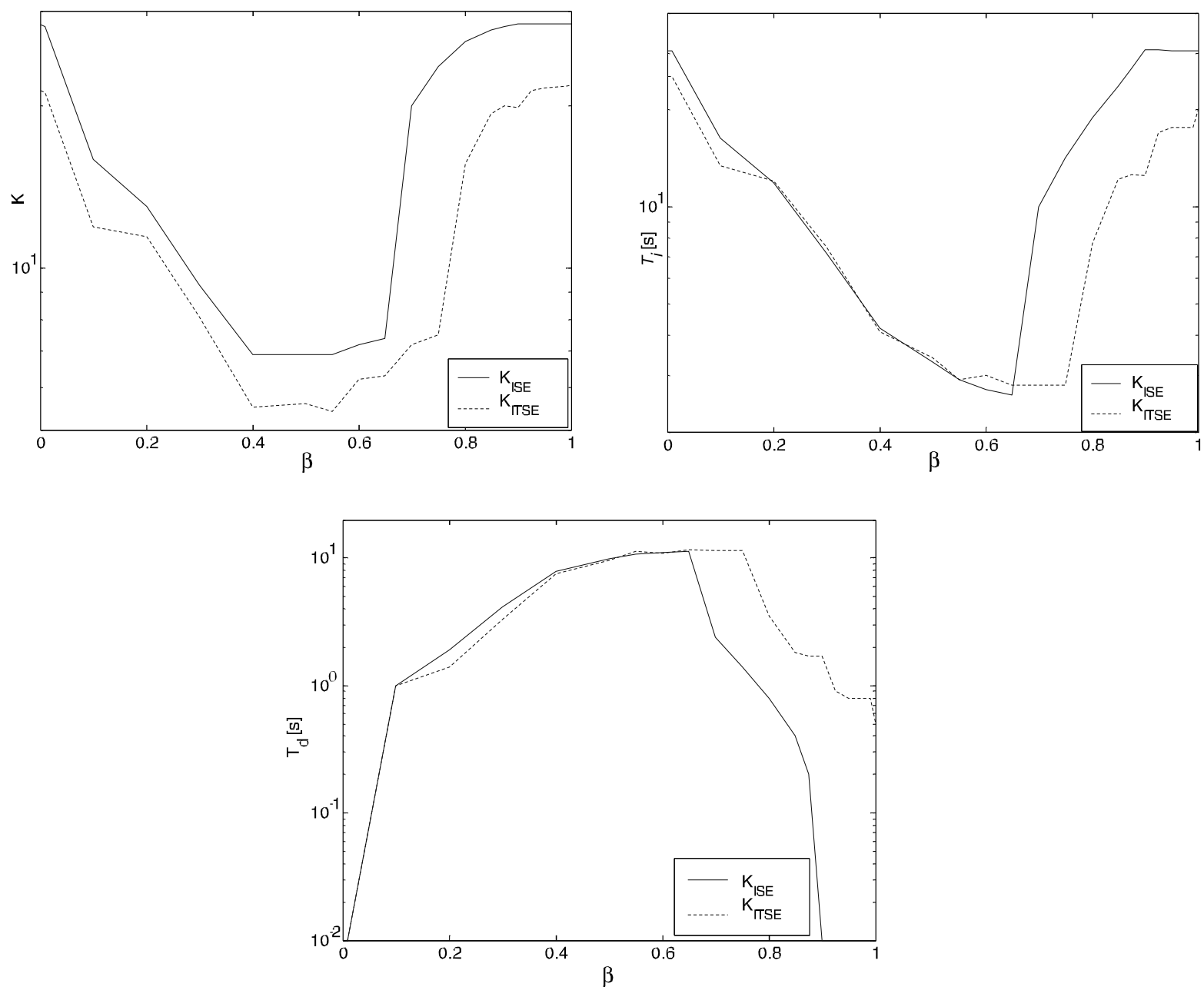

Fig. 19 The SP_PID ${ }^{\beta}$ parameters $\left(K, T_{i}, T_{d}\right)$ versus $\beta$ for the ISE and the ITSE criteria, $\delta=\infty, k=0.042 \mathrm{~m}^{2} \mathrm{~s}^{-1}$
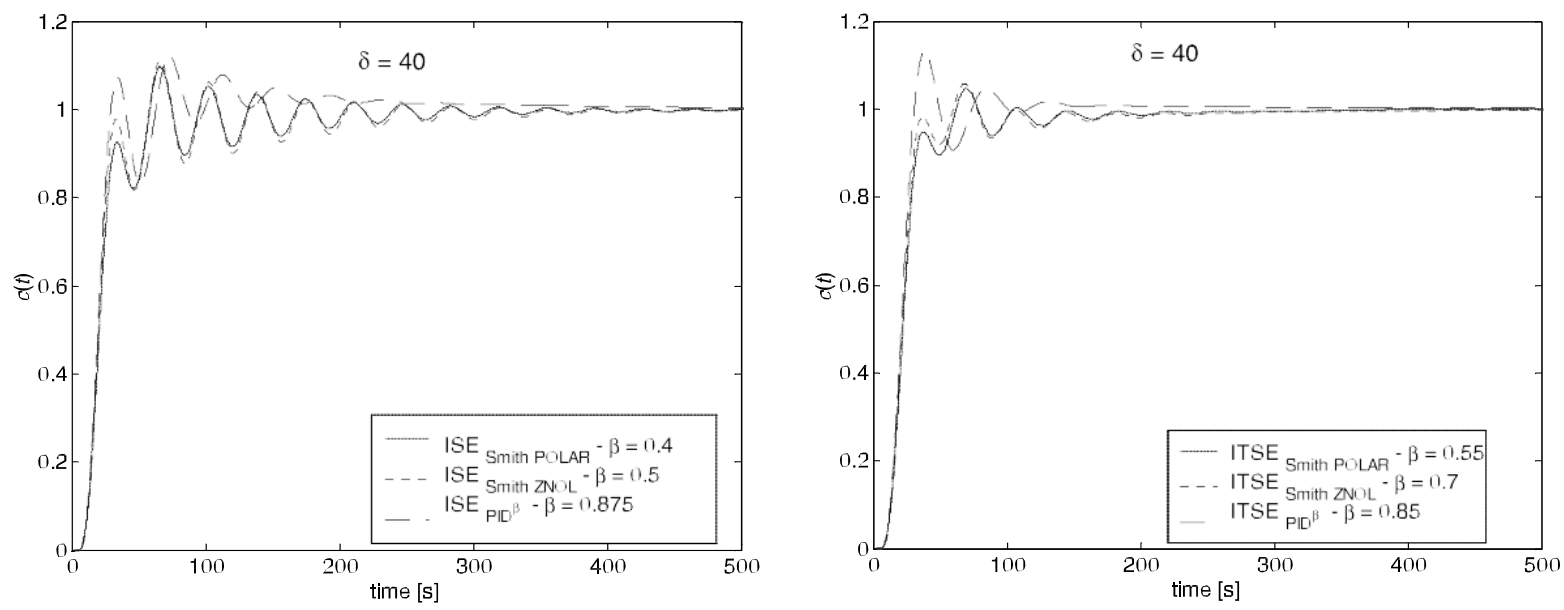

Fig. 20 Step responses of the closed-loop system for the SP_PID ${ }^{\beta}\left[\hat{G}_{\text {Polar }}\right]$, SP_PID $^{\beta}\left[\hat{G}_{\text {ZNOL }}\right]$ and PID ${ }^{\beta}$, the ISE and the ITSE indices, the optimal values of $\beta$ in all cases, $\delta=40, x=3.0 \mathrm{~m}, k=0.042 \mathrm{~m}^{2} \mathrm{~s}^{-1}$ 

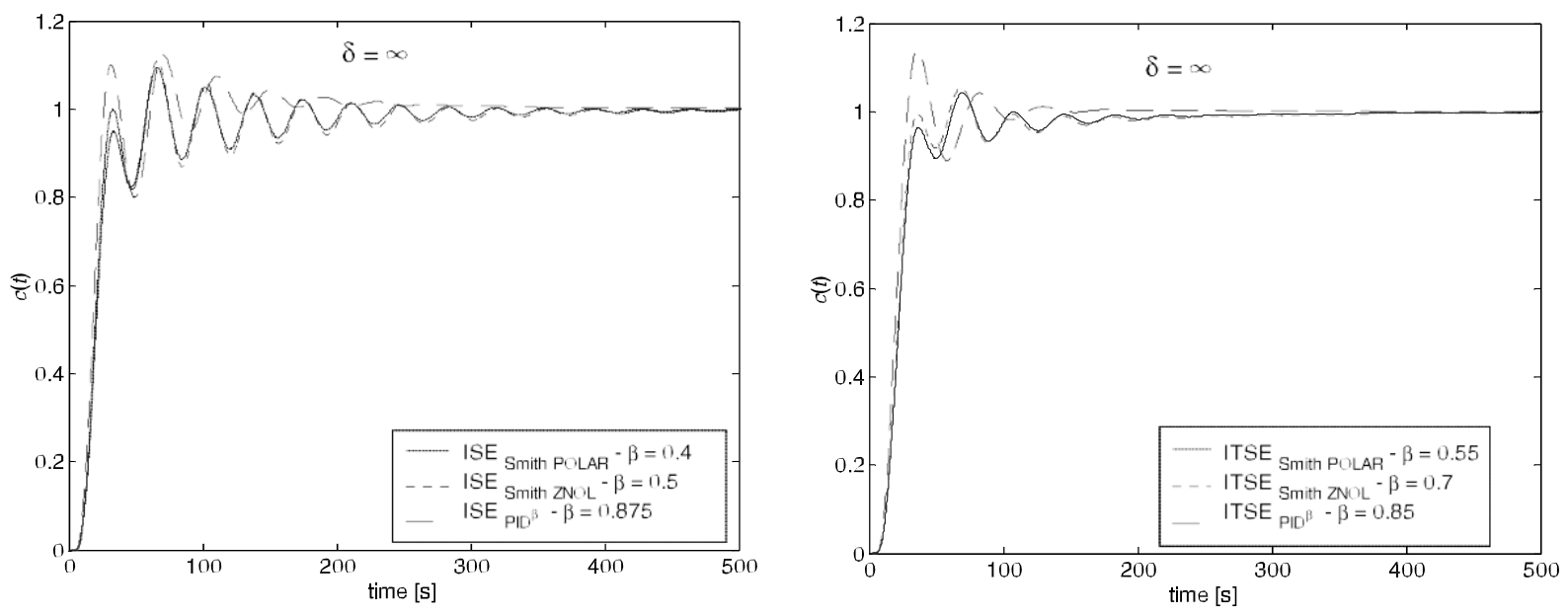

Fig. 21 Step responses of the closed-loop system for the SP_PID ${ }^{\beta}\left[\hat{G}_{P o l a r}\right]$, SP_PID $^{\beta}\left[\hat{G}_{Z N O L}\right]$ and PID ${ }^{\beta}$, for the ISE and the ITSE indices, for best $\beta$ in all cases, $\delta=\infty, x=3.0 \mathrm{~m}, k=0.042 \mathrm{~m}^{2} \mathrm{~s}^{-1}$
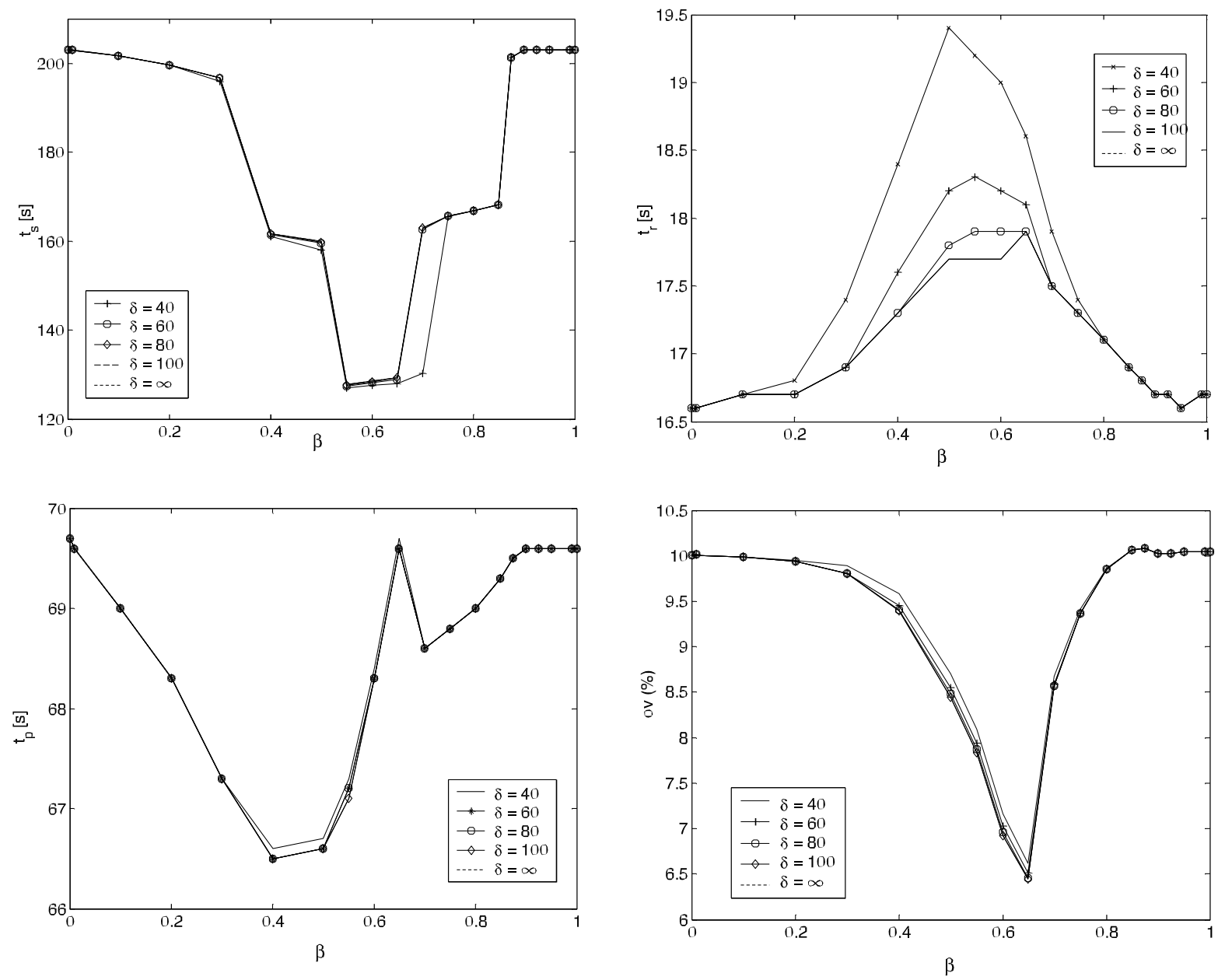

Fig. 22 Parameters $t_{s}, t_{r}, t_{p}$, ov $(\%)$ for the step responses of the closed-loop system with a SP_PID ${ }^{\beta}\left[\hat{G}_{P o l a r}\right.$, for the ISE, $\delta=\{40,60,80,100, \infty\}, x=3.0 \mathrm{~m}, k=0.042 \mathrm{~m}^{2} \mathrm{~s}^{-1}$ 

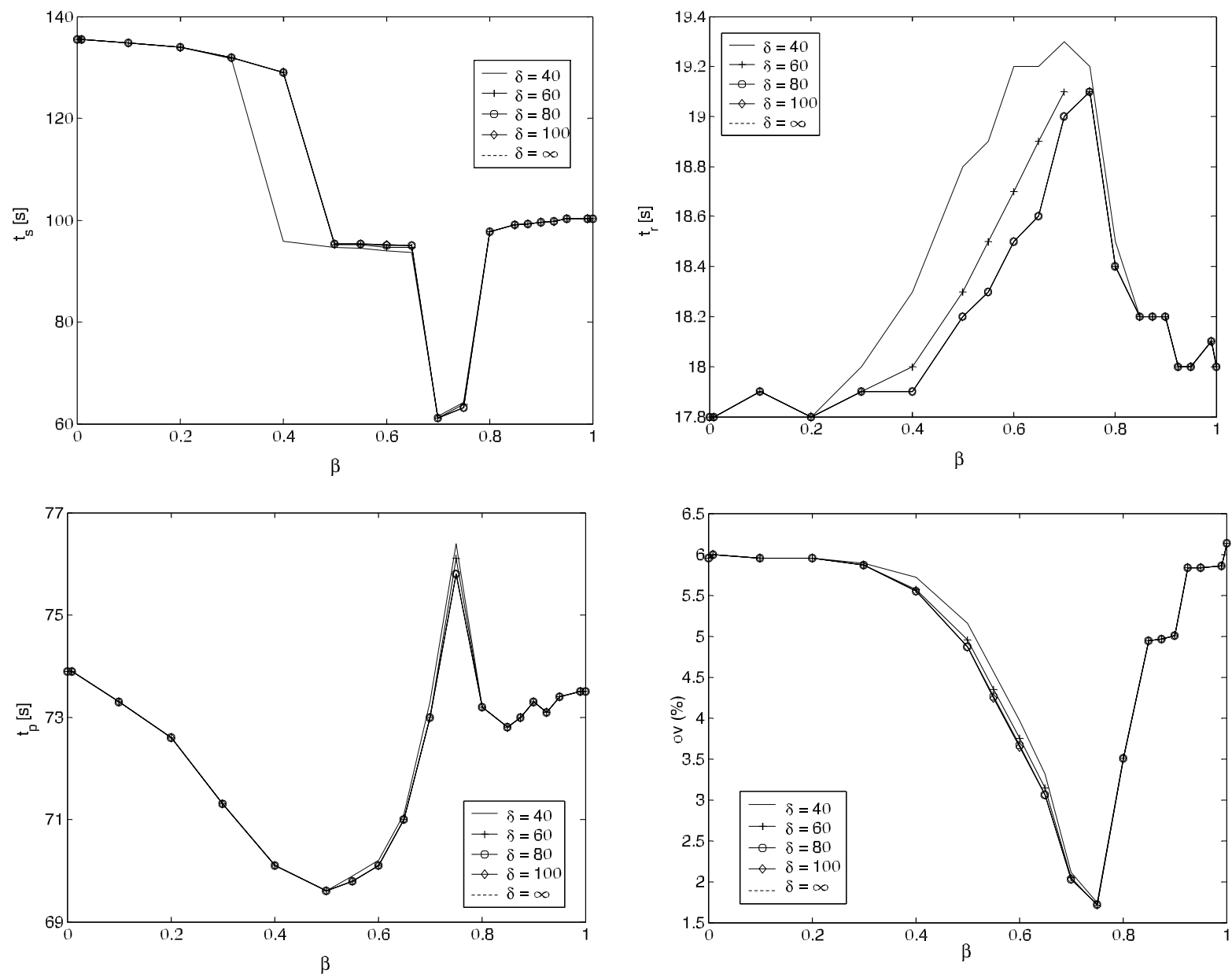

Fig. 23 Parameters $t_{s}, t_{r}, t_{p}$, ov $(\%)$ for the step responses of the closed-loop system with a SP_PID ${ }^{\beta}\left[\hat{G}_{\text {Polar }}\right]$, for the ITSE, $\delta=\{40,60,80,100, \infty\}, x=3.0 \mathrm{~m}, k=0.042 \mathrm{~m}^{2} \mathrm{~s}^{-1}$
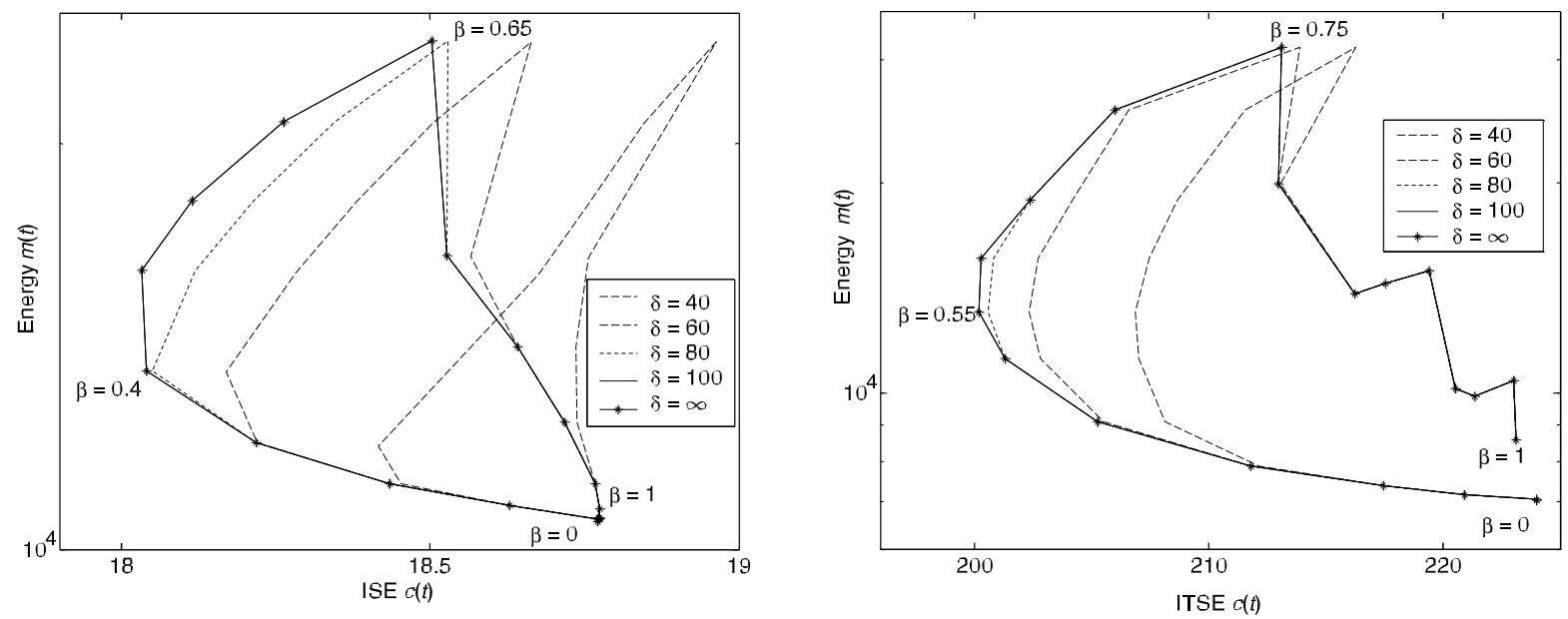

Fig. 24 Energy $E_{m}$ versus the ISE and the ITSE indices for $\delta=\{40,60,80,100, \infty\}, 0 \leq \beta \leq 1, k=0.042 \mathrm{~m}^{2} \mathrm{~s}^{-1}$ 

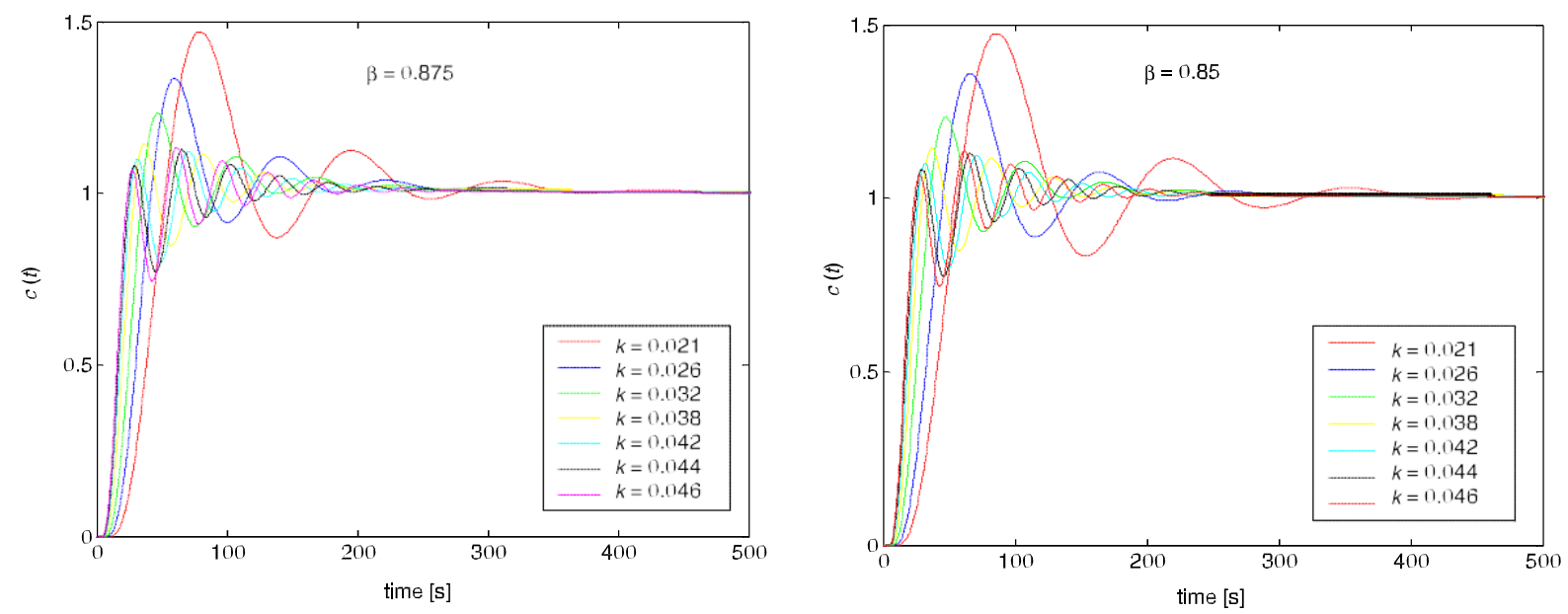

Fig. 25 Step responses of the closed-loop system for the PID $\beta$, for the ISE and the ITSE indices, $k=\{0.021,0.026,0.032$, $0.038,0.042,0.044,0.046\}, \delta=\infty, x=3.0 \mathrm{~m}$ and for $\beta_{\text {ISE }}=0.875$ and $\beta_{\text {ITSE }}=0.85$
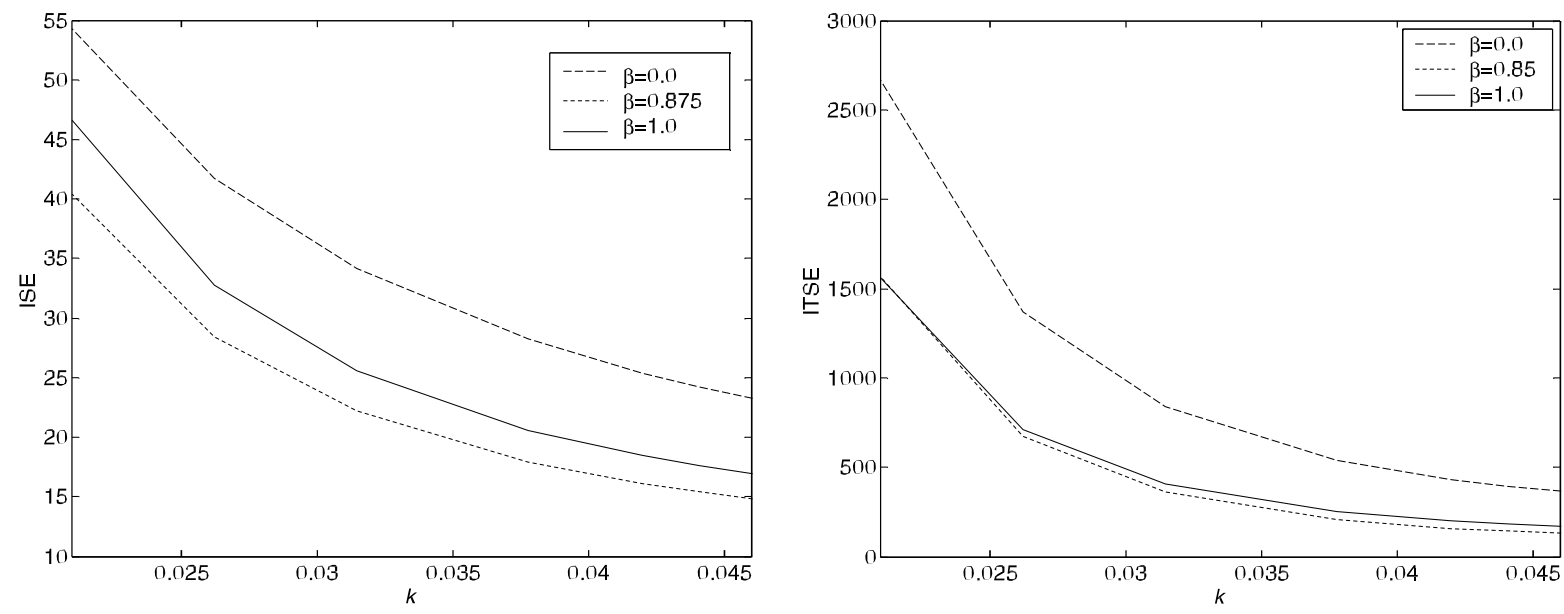

Fig. $26 k$ versus the ISE and the ITSE indices for $k=\{0.021,0.026,0.032,0.038,0.042,0.044,0.046\}, \delta=\infty, x=3.0 \mathrm{~m}$ and for $\beta_{\text {ISE }}=\{0.0,0.875,1.0\}$ and $\beta_{\text {ITSE }}=\{0.0,0.85,1.0\}$

mated through a least-squares fit between $\tilde{G}(j \omega)$ and $\hat{G}(j \omega)$ as can be seen in the polar diagram of Fig. 3:

$$
\hat{G}_{\text {Polar }}(s)=\frac{0.52}{139 s+1} e^{-28 s} .
$$

The SP_PID ${ }^{\beta}$ controller is tuned through the ISE and the ITSE criteria for $\delta=\infty$. Figure 19 illustrates the variation of the $\mathrm{PID}^{\beta}$ parameters $\left(K, T_{i}, T_{d}\right)$ as function of the order's derivative $\beta$ for the ISE and the ITSE without actuator saturation.

Figures 20 and 21 illustrate the step responses of the closed-loop system for $x=3.0 \mathrm{~m}$ when applying a step input $R(s)=1 / s$ at $x=0.0 \mathrm{~m}$. In the SP_PID $\beta$ algorithms are adopted the optimal values of $\bar{\beta}$, namely, $\beta_{\text {ISE }}=0.4$ and $\beta_{\text {ITSE }}=0.55$ for the SP_PID ${ }^{\beta}\left[\hat{G}_{\text {Polar }}\right], \beta_{\text {ISE }}=0.5$ and $\beta_{\text {ITSE }}=0.7$ for the SP_PID $\beta\left[\hat{G}_{Z N O L}\right]$, and $\beta_{\text {ISE }}=0.875$ and $\beta_{\text {ITSE }}=$ 0.85 for the $\mathrm{PID}^{\beta}$, respectively. For both criteria, we represent the time responses for $\delta=40$ and $\delta=\infty$.

Once again the graphs show a better transient response for the simpler $\mathrm{PID}^{\beta}$ controller, namely, smaller values of the $t_{s}$, while the $t_{d}$ and $t_{r}$ are approximately identical, for all cases. 

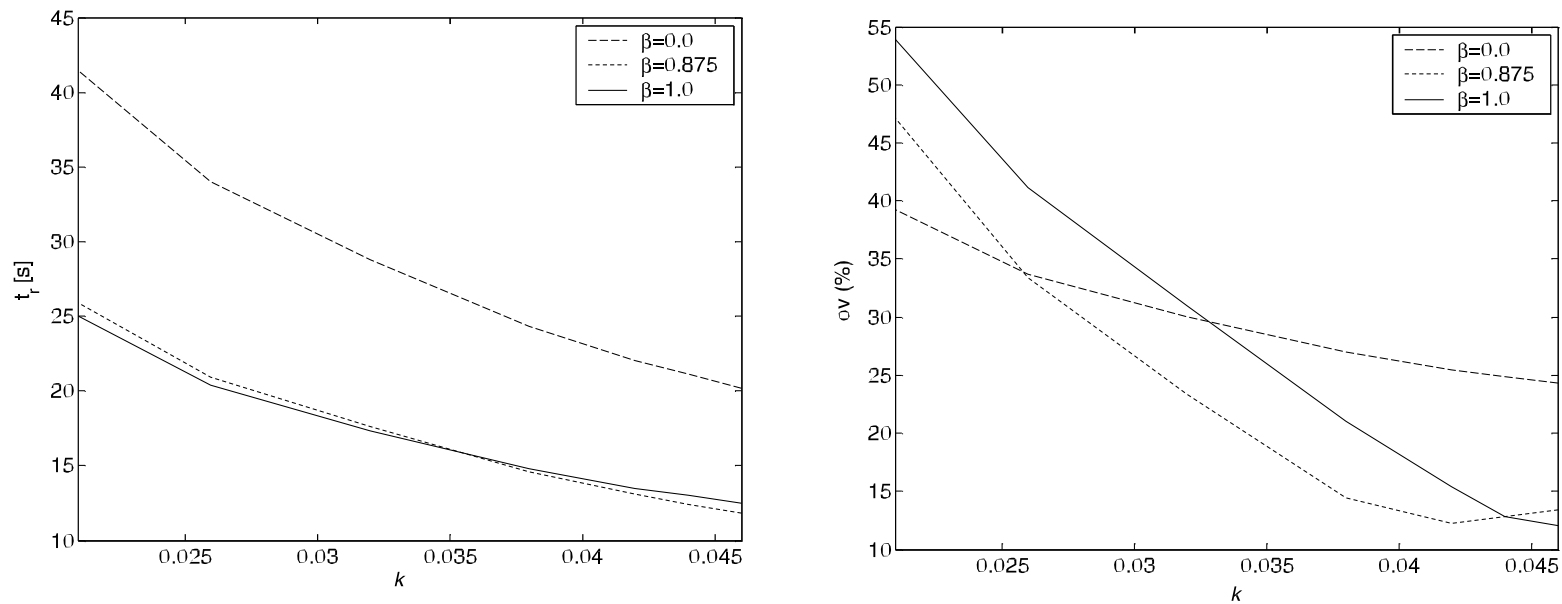

Fig. 27 Parameters $t_{r}, o v(\%)$ for the step responses of the closed-loop system with a PID ${ }^{\beta}$, for the ISE, $\delta=\infty, x=3.0 \mathrm{~m}$ and for $\beta=\{0.0,0.875,1.0\}$
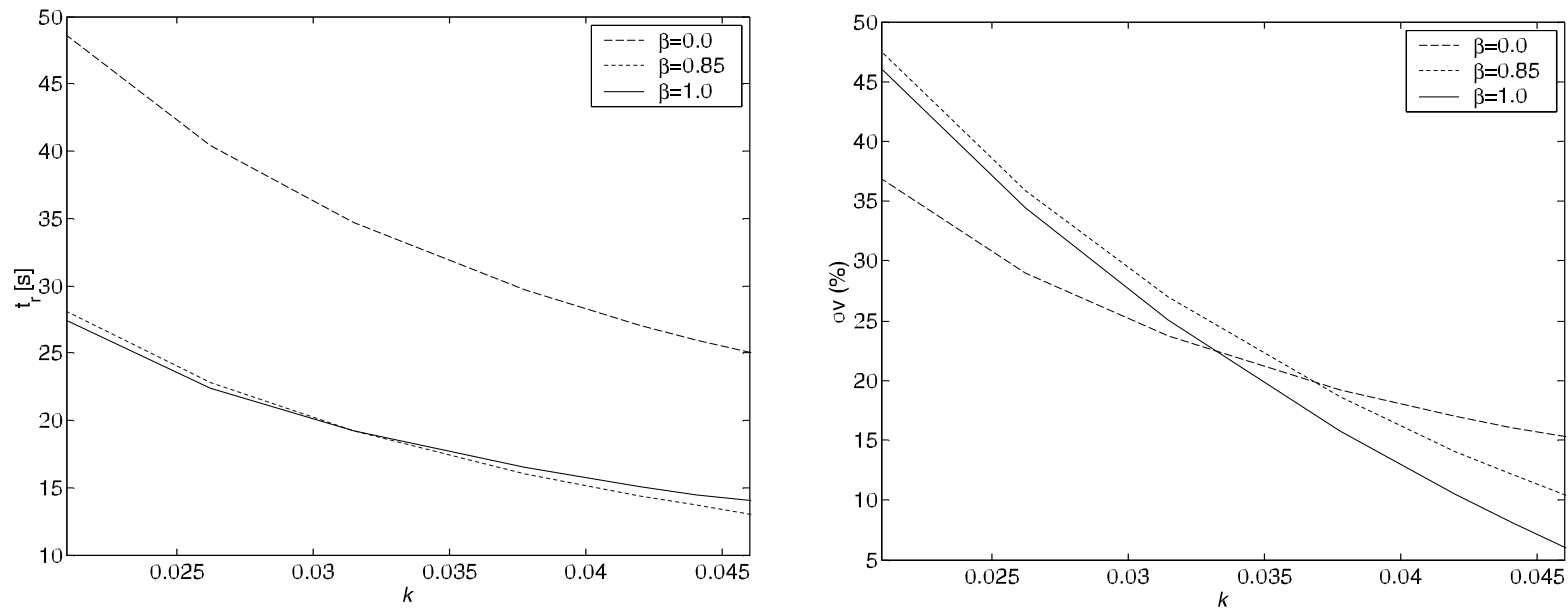

Fig. 28 Parameters $t_{r}, o v(\%)$ for the step responses of the closed-loop system with a PID ${ }^{\beta}$, for the ITSE, $\delta=\infty, x=3.0 \mathrm{~m}$ and for $\beta=\{0.0,0.85,1.0\}$

Figures 22 and 23 depict the variation of the transient response parameters $\left\{t_{s}, t_{r}, t_{p}, o v(\%)\right\}$ for the closed-loop response with the SP_PID ${ }^{\beta}\left[\hat{G}_{\text {Polar }}\right]$ tuned thought the minimization of the ISE and the ITSE indices, respectively.

The best compromise values for the distinct region in the charts is $\beta_{\text {ISE }} \approx 0.4$ and $\beta_{\text {ITSE }} \approx 0.55$.

Figure 24 depicts the relation between the energy $E_{m}$ and the ISE and ITSE indices. We verify that the best case is achieved when $\left(\beta_{\text {ISE }}, \beta_{\text {ITSE }}\right)=$ $(0.4,0.55)$, revealing that with a SP_PID ${ }^{\beta}\left[\hat{G}_{\text {Polar }}\right]$, the effectiveness of the $\mathrm{PID}^{\beta}$ is superior to the case of adopting an inferior model $\hat{G}_{Z N O L}$.
In the case of control using SP, the two adopted models proved to be insufficient to get superior performance. Therefore, for an efficient use of the SP a better approximation model of the heat system should be envisaged. In this line of thought, the adoption of a fractional order model $\hat{G}(s)$ will be addressed in future research.

4.5 The PID ${ }^{\beta}$ versus the diffusivity constant $k$

In this subsection, we analyze the performance of fractional $\mathrm{PID}^{\beta}$ controller, proposed in the previous 
Sect. 4.2 for systems with different values of the diffusivity constant $k$.

A step reference input $R(s)=1 / s$ is applied at $x=$ $0.0 \mathrm{~m}$ and the output $c(t)$ is analyzed for $x=3.0 \mathrm{~m}$ without considering actuator saturation. We adopt the $\mathrm{PID}^{\beta}$ parameters obtained in the Sect. 4.2 for the ISE and for the ITSE indices, namely, ISE: $\left\{K, T_{i}, T_{d}, \beta\right\} \equiv\{3,23,90.6,0.875\}$ and ITSE: $\left\{K, T_{i}, T_{d}, \beta\right\} \equiv\{1.8,17.6,103.6,0.85\}$.

Figure 25 depicts the step responses of the closedloop system for different values of diffusivity constant, namely, $k=\{0.021,0.026,0.032,0.038,0.042,0.044$, $0.046\}$.

The step responses of the $\mathrm{PID}^{\beta}$ reveal a large diminishing of the overshoot and the rise time when $k$ decreases. All responses show a good transient and a zero steady-state error. Once more, these results demonstrate the effectiveness of the fractional algorithms when used for the control of diffusion systems.

Figure 26 illustrates the variation of the ISE and the ITSE criteria with $k$, under the action of $\mathrm{PID}^{\beta}$, for $\beta_{\text {ISE }}=\{0.0,0.875,1.0\}$ and $\beta_{\text {ITSE }}=\{0.0,0.85$, $1.0\}$. The figures reveal that the values of the ISE and the ITSE diminish when $k$ increases. Moreover, the best results occur for the fractional PID.

Figures 27 and 28 show the variation of $t_{r}$ and $o v$ (\%) versus $\beta$ for the same conditions of Fig. 26. The charts of $t_{r}$ reveal for both indices similar patterns. The results for $\beta=0.0$ are clearly the worst. For the other two cases, $t_{r}$ diminishes with $k$, and for $k>0.04$, the fractional algorithm produces better results.

For the $o v(\%)$, we verify that for the ISE, the fractional algorithm presents the best results. For the ITSE case, we get the same behavior, nevertheless, the integer PID leads to the best solution for large values of $k$.

In conclusion, these results demonstrate the effectiveness of the fractional-order algorithms when used for the control of diffusion systems.

\section{Conclusions}

This paper presented the fundamental aspects of application of the FC theory in the control of diffusion systems. In this line of thought, a heat diffusion system was studied and described through the fractional-order operator $s^{0.5}$. The dynamics of the system were analyzed in the perspective of FC, and some of its implications upon the control algorithms and systems with time delay were investigated.

We presented four kinds of control strategies, namely, integer and fractional order controllers, and a Smith Predictor with two types of first order models. We concluded that with a PID $^{\beta}$ controller we can get better results than those obtained with the SP. This result points out the use of the fractional PID algorithm instead of SP because the controller is simple and easier to implement.

\section{References}

1. Oldham, K.B., Spanier, J.: The Fractional Calculus: Theory and Application of Differentiation and Integration to Arbitrary Order. Academic Press, New York (1974)

2. Podlubny, I.: Fractional Differential Equations. Academic Press, San Diego (1999)

3. Battaglia, J.L., Cois, O., Puigsegur, L., Oustaloup, A.: Solving an inverse heat conduction problem using a non-integer identified model. Int. J. Heat Mass Transf. 44, 2671-2680 (2001)

4. Courant, R., Hilbert, D.: Methods of Mathematical Physics, Partial Differential Equations. Wiley-Interscience II, New York (1962)

5. Podlubny, I.: Fractional-order systems and $\mathrm{PI}^{\lambda} \mathrm{D}^{\mu}$ controllers. IEEE Trans. Automat. Contr. 44(1), 208-213 (1999)

6. Vinagre, B.M., Chen, Y.Q., Petráš, I.: Two direct Tustin discretization methods for fractional-order differentiator/integrator. Franklin Inst. 340(5), 349-362 (2003)

7. Chen, Y.Q., Vinagre, B.M., Podlubny, I.: Continued fraction expansion to discretize fractional order derivativesan expository review. Nonlinear Dyn. 38(1-4), 155-170 (2004)

8. Barbosa, R.S., Tenreiro Machado, J.A., Silva, M.F.: Time domain design of fractional differintegrators using leastsquares. Signal Process. 86(10), 2567-2581 (2006)

9. Zhuang, M., Atherton, D.P.: Automatic tuning of optimum PID controllers. IEE Proc., Control Theory Appl. 140(3), 216-224 (1993)

10. Petrás, I., Vinagre, B.M.: Practical application of digital fractional-order controller to temperature control. Acta Montan. Slovaca 7(2), 131-137 (2002)

11. Barbosa, R.S., Tenreiro Machado, J.A., Ferreira, I.M.: Tuning of PID controllers based on Bode's ideal transfer function. Nonlinear Dyn. 38(1/4), 305-321 (2004)

12. Chen, Y.Q., Moore, K.L.: Relay feedback tuning of robust PID controllers with iso-damping property. IEEE Trans. Syst. Man Cybern., Part B, Cybern. 35(1), 23-31(2005)

13. Tenreiro Machado, J., Jesus, I., Boaventura Cunha, J., Tar, J.K.: Fractional Dynamics and Control of Distributed Parameter Systems. In: Intelligent Systems at the Service of Mankind, Vol. 2, pp. 295-305. Ubooks (2006) 
14. Crank, J.: The Mathematics of Diffusion. Oxford University Press, London (1956)

15. Gerald, C.F., Wheatley, P.O.: Applied Numerical Analysis. Addison Wesley, Reading (1999)

16. Farlow, S.J.: Partial Differential Equations for Scientists and Engineers. Wiley, New York (1993)

17. Chen, Y.Q.: Ubiquitous fractional order controls? In: The Second IFAC Symposium on Fractional Derivatives and Applications-IFAC-FDA06, July, Portugal (2006)
18. Jesus, I.S., Barbosa, R.S., Tenreiro Machado, J.A., Boaventura Cunha, J.: Strategies for the control of heat diffusion systems based on fractional calculus. In: IEEE-ICCC 2006-IEEE International Conference on Computational Cybernetics, August 3-8, Estonia (2006)

19. Smith, O.J.M.: Closed control of loops with dead time. Chem. Eng. Process. 53, 217-219 (1957) 